\title{
Performance comparison of cooperative relay links with different relay processing strategies: Nakagami/Gamma approximation approaches
}

\author{
Jia Shi ${ }^{*}$, Chen Dong and Lie-Liang Yang
}

\begin{abstract}
In this article, we investigate and compare the error performance of two-hop communication links (THCLs) with multiple relays, when distributed and cooperative relay processing schemes are respectively employed. Our main objectives include finding some general and relatively simple ways for estimating error performance and demonstrating the trade-off of using cooperative relay processing. One distributed relay processing and two cooperative relay processing schemes are compared. In the two cooperative relay processing schemes, one assumes the ideal relay cooperation, in which relays exchange information without consuming energy, while the other one assumes energy consumption for relay cooperation. In this paper, the error performance of the THCLs employing the considered relay processing schemes is investigated, when the channels from source to relays, the channels for information exchange and that from relays to destination experience various types of fading modeled by the Nakagami- $m$ distributions. In order to derive the formulas for the bit error rate (BER) of the THCLs employing binary phase-shift keying (BPSK) modulation and various relay processing schemes, we introduce the Nakagami and Gamma approximation for finding the distribution functions of various variables encountered. Our studies show that the proposed approximation approaches are highly effective, which are capable of accurately predicting the BER of the THCLs supported by the different relay processing schemes.
\end{abstract}

Keywords: Cooperative communications; Relay communications; Distributed processing; Transmitter preprocessing; Maximal-ratio combining; Equal gain combining; Nakagami fading

\section{Introduction}

It has been widely recognized that cooperative communications will play important roles in the future generations of wireless communication systems [1-4]. One type of cooperative communication systems is the relayassisted, where distributed mobile nodes, often referred to as relays, are employed for attaining cooperative diversity, in order to enhance the reliability of wireless communications $[5,6]$. The relay-assisted wireless communication systems have been investigated in the context of various relay protocols, which include amplify-and-forward (AF), decode-and-forward (DF), compress-and-forward (CF) protocols, etc. [5-7].

*Correspondence: js22g09@ecs.soton.ac.uk

1 School of Electronics and Computer Science, University of Southampton, Southampton, SO17 1BJ, UK
Along with the relay-assisted wireless communications, a lot of researches have addressed the bit error rate (BER) or symbol error rate (SER) analysis, when assuming communications over, such as, Rayleigh fading, Rician fading and Nakagami- $m$ fading channels [8-15]. In the analyses, various cooperative relay scenarios have been considered, which include the classic three-node relay network $[13,14]$, serial or parallel multihop cooperative relay networks $[9,12,15]$, etc. A lot of exact or approximate closed-form formulas have been derived for evaluating the BER/SER of considered scenarios. In [10], the exact average SER formulas have been obtained for the cooperative network, where a source sends messages to a destination with the aid of multiple AF relays, when assuming communications over flat Rayleigh fading channels. In [11], the SER analysis has been done in the context of the multihop cooperative relay networks over various types of 
fading channels, when both the number of relays and the number of hops may take arbitrary values.

In addition to BER/SER, the outage probability of cooperative wireless systems has been investigated, for example, in [16-19]. In more detail, the lower and asymptotic bounds of outage probability have been derived in [16] for dual-hop relay networks experiencing Rayleigh fading. By contrast, in $[17,19]$, the outage probability has been derived, when assuming communication over independent and non-identically distributed Nakagami- $m$ fading channels. Furthermore, considering capacity/throughput, in [20-22], the authors have studied the capacity bound and rate region of two-hop relay networks. In [21], a traditional three-node relay system has been considered, and the upper and lower bounds of ergodic capacity have been derived, when various relay schemes are employed. In [22], the capacity bounds have been analyzed in the context of multinode ad hoc networks.

In the published references, such as $[13,18,23,24]$, on the relay communications employing cooperative relays, a typical assumption used is ideal cooperation among relays. Under this assumption, there is no energy consumption for the information exchange required by cooperation and, furthermore, other overheads required are also often ignored.

Against this background, in [25], we studied and compared various relay processing schemes in association with the two-hop communication links (THCLs), where multiple relays assisted one source to communicate with a destination. We demonstrated that the studies under ideal assumptions often result in misleading observations, when practical scenarios were considered. Continuing the work in [25], in this paper, we investigate further three types of relay processing schemes. The first one is the distributed relay processing (DRP), in which relays do not cooperate with each other, whereas process their signals independently in a distributed way to achieve the transmitter equal-gain combining (TEGC). The second scheme is the ideal centralized maximal ratio combining (MRC) and TEGC relay processing, which is termed as ICRP. In this scheme, signals received by the relays from the source are ideally forwarded to a so-called information exchange central unit (IECU) without consuming any energy. After the MRC-assisted detection at the IECU, the IECU broadcasts the detected data back to the relays also without consuming any energy and without error. Finally, the third scheme is the centralized MRC and TEGC relay processing, which is referred to as CRP and has the same structure as the ICRP, except the assumptions for information exchange. Specifically, after the relays receive the signals from the source, they convey the information to the IECU based on the principles of direct-sequence code-division multiple-access (DS-CDMA). After the IECU detects the signals, it broadcasts the information back to the relays also over non-ideal channels. The system needs to allocate energy for implementation of both the above processes.

In this paper, assuming binary phase-shift keying (BPSK) baseband modulation, we analyze the BER of the THCLs employing the above-stated various relay processing schemes, when assuming that the source-to-relay (S-R) channels, the multiple-access (MA) channels from relays to IECU, the broadcast $(\mathrm{BC})$ channels from IECU to relays, and the relay-to-destination (R-D) channels experience independent flat Nakagami- $m$ fading. As the exact BER analyses for the considered scenarios are extremely hard - if they are not impossible, we propose some general and accurate approximation approaches for reaching our objectives. Specifically, the proposed approximation approaches include the Nakagami theoretical approximation (Nakagami-TAp), the Nakagami statistical approximation (Nakagami-SAp), and the Gamma approximation (Gamma-Ap). The principles of these approaches as well as their applications will become explicit in our forthcoming discourses. Finally, the error performance of the various relay processing schemes are demonstrated and compared based on the results obtained by both simulations and evaluation of the formulas analytically obtained. The results show that the error performance predicted from the formulas derived based on the approximation approaches agree well with that obtained by simulations for the various scenarios addressed.

The rest of the paper is organized as follows: Section 2 states the system model. Section 3 details the relay processing schemes. In Section 4, we analyze the average BER of the THCLs employing various relay processing schemes. Section 5 demonstrates the BER performance of the THCL systems. Finally, in section 6 , we summarize our main findings.

\section{System model}

Figure 1 is the schematic diagram for the THCL system considered in this paper. As shown in Figure 1, a THCL consists of one source, one destination and $L$ relays. Information is transmitted from the source to the destination with the aid of the $L$ relays, which either cooperate with each other or independently process their signals. Furthermore, when the cooperative relay processing schemes (ICRP and CRP) are employed, information exchange among relays are accomplished via an IECU, as shown in Figure 1.

We assume that each of the communication terminals, including the source, destination, relays, and IECU, is equipped with one antenna for signal receiving and transmission. The source and destination are separated by a long distance and unable to communicate directly. Hence, information is transmitted from source to destination in two hops under the support of relays. We assume that the $L$ relays from a cluster and are close to each other. 


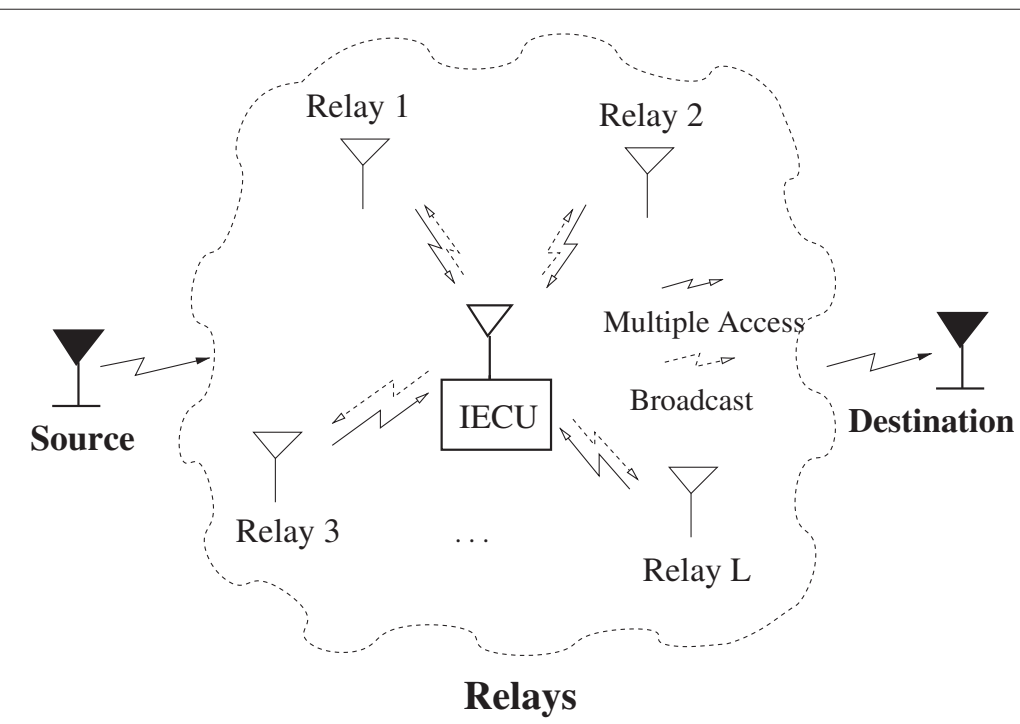

Figure 1 Schematic diagram for the two-hop communication links.

When the relays are operated in cooperation mode, we assume that the IECU seats in the middle of the $L$ relays and has small and similar distances from all the $L$ relays. We also assume that the relays do not communicate with each other, instead, they receive signals from the source, share their information with the aid of the IECU and independently process and transmit their signals to the destination. By contrast, the IECU is assumed to communicate only with the relays, it does not receive signals from the source or transmit signals to the destination. Note that the IECU may be viewed as a signal processing unit, which implements multi-way relay $[26,27]$ to aid information exchange among relays. As shown in Figure 1, the relays forward their signals to the IECU based on the principles of DS-CDMA and, then, the IECU broadcasts the processed signal back to the relays. In this paper, we assume that all communication terminals are operated in half-duplex mode. We assume that a relay employs the channel state information (CSI) of both the S-R and R-D channels related to this relay. The IECU has the CSI required for carrying out MRC. Furthermore, when the CRP is employed, a relay is also assumed to have the CSI of the IECU's BC channel to this relay.

Let the source transmit a symbol $x$, which satisfies $E[x]=0$ and $E\left[|x|^{2}\right]=1$. Then, the received observations by the $L$ relays can be expressed in vector form as

$$
\boldsymbol{y}_{r}=\sqrt{\alpha_{1}} \boldsymbol{h}_{\mathrm{sr}} \boldsymbol{x}+\boldsymbol{n}_{r}
$$

where $\boldsymbol{y}_{r}=\left[y_{r_{1}}, y_{r_{2}}, \ldots, y_{r_{L}}\right]^{T}$ and $y_{r_{i}}$ represents the observation of the $i$ th relay, $\boldsymbol{h}_{\mathrm{sr}}=\left[h_{\mathrm{sr}_{1}}, h_{\mathrm{sr}_{2}}, \ldots, h_{\mathrm{sr}_{L}}\right]^{T}$ contains the channel gains of the $L \mathrm{~S}-\mathrm{R}$ channels, $\alpha_{1}$ depends on the relative power allocated to the first hop, and $\boldsymbol{n}_{r}=$ $\left[n_{r_{1}}, n_{r_{2}}, \ldots, n_{r_{L}}\right]^{T}$ is a length- $L$ additive white Gaussian noise (AWGN) vector, each element of which obeys the complex Gaussian distribution with zero mean and a variance of $2 \sigma^{2}$, where $\sigma^{2}=1 /\left(2 \bar{\gamma}_{s}\right)$ with $\bar{\gamma}_{s}$ denoting the average sound-to-noise ratio (SNR) per symbol. From (1) we are implied that the average SNR of the first hop is $\gamma_{\text {sr }}=\alpha_{1} \bar{\gamma}_{s}$ per relay.

Based on (1), the $L$ relays carry out one of the three relay processing schemes, including the DRP, ICRP and the CRP. Note that, when the ICRP and CRP are considered, the relays use AF to send signals to the IECU. For communications between relays and destination, the DRP scheme achieving TEGC is always used to forward information to the destination, no matter which of the three relay processing schemes is employed.

Regardless of which relay processing scheme is employed, let us express the signals to be transmitted by the relays to the destination as $\tilde{\boldsymbol{y}}_{r}=\left[\tilde{y}_{r_{1}}, \tilde{y}_{r_{2}}, \ldots, \tilde{y}_{r_{L}}\right]^{T}$. Then, the received signals at the destination is in the form of

$$
y_{d}=\sum_{i=1}^{L} \sqrt{\alpha_{2}} h_{r_{i} d} \tilde{y}_{r_{i}}+n_{d}
$$

where $h_{r_{i} d}$ represents the gain of the $i$ th R-D channel, $n_{d}$ is the Gaussian noise added at the destination, which is distributed with zero mean and a variance of $2 \sigma^{2}$, while $\alpha_{2}$ is determined by the relative power allocated to the second hop. Based on (2), we can know that the average SNR of the $i$ th, where $i=1,2, \ldots, L$, R-D channel is $\gamma_{r_{i} d}=\alpha_{2} E\left[\left|\tilde{y}_{r_{i}}\right|^{2}\right] \bar{\gamma}_{s}$, where $\bar{\gamma}_{s}$ again denotes the average SNR per symbol.

Note that, for the sake of comparison of the various relay processing schemes, the total transmission power of a symbol is constraint to $P=1$, regardless of 
using distributed or cooperative relay processing, and of the number of relays. If distributed relay processing is employed, the power allocated to the first and second hops is $\alpha_{1}$ and $\alpha_{2}$, respectively, which satisfy $\alpha_{1}+\alpha_{2}=1$. By contrast, if the THCL system employs cooperative relay processing, a portion of power, which is expressed as $\alpha_{r}$, has to be allocated for information exchange among the relays. Furthermore, according to our previous discussion associated with Figure 1, information exchange requires both the MA transmission and $\mathrm{BC}$ transmission. Their corresponding power is expressed as $\alpha_{\mathrm{ma}}$ and $\alpha_{\mathrm{bc}}$, respectively. Consequently, the relationships of $\alpha_{r}=\alpha_{\mathrm{ma}}+\alpha_{\mathrm{bc}}$ and $\alpha_{1}+\alpha_{2}+\alpha_{r}=1$ are satisfied.

In our forthcoming performance analysis, we assume that the S-R channels, MA/BC channels and the R-D channels experience the generalized Nakagami- $m$ fading associated with the different parameters, which determine the fading severity. The probability density function (PDF) of the Nakagami- $m$ distribution is [28]

$$
f_{\left|h_{i j}\right|}(r \mid m, \Omega)=\frac{2 m^{m} r^{2 m-1}}{\Gamma(m) \Omega^{m}} e^{-(m / \Omega) r^{2}}, r \geq 0
$$

where the subscripts $i, j$ associated with $h_{i j}$ are dependent on the specific channel considered. In (3), $\Omega=E\left[\left|h_{i j}\right|^{2}\right]$ denotes the average power of the channel and $m(m \geq$ $0.5)$ is the Nakagami- $m$ fading parameter characterizing the severity of fading, the fading becomes less severe when the value of $m$ increases.

We also consider the special scenarios, where the MA/BC channels only suffer from AWGN, in order to demonstrate that, even in this over-optimistic communication environments, the energy spent for relay cooperation may significantly degrade the achievable performance.

\section{Relay processing}

In this article, three types of relay processing schemes are investigated, which include (1) DRP: TEGC-assisted distributed relay processing; (2) ICRP: ideal centralized MRC- and TEGC-aided relay processing, which implements ideal relay cooperation; and (3) CRP: centralized MRC- and TEGC-aided relay processing, which requires energy for carrying out relay cooperation. Regardless of which of the above three schemes is employed, we assume that the DRP achieving TEGC is employed by the relays for forwarding information to the destination. For this sake, below, we first discuss the principles of the DRP.

\subsection{DRP}

During the R-D transmission, we assume that every relay has the CSI of the channel between it and the destination. Since there is no cooperation among relays, the relays can only carry out distributed transmitter preprocessing; every relay can only try to maximize the SNR of the link between it and the destination, which is optimum when considering the individual links. At the receiver, equalgain combining (EGC) is achieved and, therefore, we have the TEGC-assisted DRP, which is simply referred to as DRP. Explicitly, the TEGC-assisted DRP is optimum in the sense of maximizing the SNR at the destination.

Under the DRP, the signal forwarded by the $i$ th relay to the destination is given by

$$
\tilde{y}_{r_{i}}=\frac{1}{\sqrt{L}} \frac{h_{r_{i} d}^{*}}{\sqrt{\left|h_{r_{i} d}\right|^{2}}} x_{r_{i}}, \quad i=1,2, \ldots, L
$$

where $x_{r_{i}}$ is the symbol detected by the $i$ th relay based on the observation (1), while $1 / \sqrt{L}$ is for satisfying the power constraint of the R-D channels. Consequently, after substituting (4) into (2), the decision variable formed by the destination is

$$
y_{d}=\sum_{i=1}^{L} \sqrt{\frac{\alpha_{2}}{L}}\left|h_{r_{i} d}\right| x_{r_{i}}+n_{d}
$$

Explicitly, the diversity order achieved by the DRP is $L$.

When BPSK is employed and when assuming that there are $l$ relays (with the indices $\left.i \in{ }_{l}\{1, \ldots, L\}\right)$ making correct detection, while the remaining $q=L-l$ relays (having the indices $\left.j \hat{\epsilon}_{q}\{1, \ldots, L\}\right)$ make erroneous detection, where $\epsilon_{l}\{1, \ldots, L\}$ represents selecting $l$ numbers from $\{1, \ldots, L\}$, while $\hat{\epsilon}_{q}\{1, \ldots, L\}$ means the remaining $q$ numbers of $\{1, \ldots, L\}$ after the selections. Then, the decision variable of (5) can be written as

$$
\begin{aligned}
y_{d}^{(l, q)} & =\sqrt{\frac{\alpha_{2}}{L}}\left[\sum_{i \in l\{1, \ldots, L\}}\left|h_{r_{i} d}\right|-\sum_{j \hat{\epsilon}_{q}\{1, \ldots, L\}}\left|h_{r_{j} d}\right|\right] x+n_{d} \\
& =\sqrt{\frac{\alpha_{2}}{L}}\left[h_{\sum l}-h_{\sum q}\right] x+n_{d} \\
& =\sqrt{\frac{\alpha_{2}}{L}} h_{l, q} x+n_{d}
\end{aligned}
$$

where, for convenience of BER analysis, we defined $h_{\sum l}=$ $\sum_{i \in\{\{1, \ldots, L\}}\left|h_{r_{i} d}\right|, h_{\sum q}=\sum_{j \hat{\epsilon}_{q}\{1, \ldots, L\}}\left|h_{r_{j} d}\right|$ and $h_{l, q}=$ $h_{\sum l}-h_{\sum q}$.

\subsection{ICRP}

When the ICRP is employed, we assume that information exchange among the $L$ relays is ideal (error-free) and does not consume energy. This is a typical assumption used in many references, such as in $[13,18,23,24]$, considering cooperative relays. In this case, the total power $P=1$ is only consumed by the first (S-R) and second (RD) hops. Therefore, we have $\alpha_{1}+\alpha_{2}=1$. At the IECU, the symbol transmitted by the source is detected with the aid of MRC. Then, the IECU returns the detected symbol to the relays without consuming energy. Finally, after the TEGC-assisted preprocessing, the relays forward the symbol received from the IECU to the destination. 
Since information exchange is ideal, the signals received by the IECU are given by $\boldsymbol{y}_{\mathrm{cu}}=\boldsymbol{y}_{r}$, where $\boldsymbol{y}_{r}$ is given by (1). In this case, assuming that the CSI of all S-R channels are known to the IECU, it can hence form the decision variable based on MRC as

$$
z_{\mathrm{cu}}=\boldsymbol{h}_{\mathrm{sr}}^{H} \boldsymbol{y}_{\mathrm{cu}}=\sum_{i=1}^{L}\left(\sqrt{\alpha_{1}}\left|h_{\mathrm{sr}_{i}}\right|^{2} x+h_{\mathrm{sr}_{i}}^{*} n_{r_{i}}\right)
$$

From (7), we can know that the instantaneous SNR can be expressed as

$$
\gamma_{\mathrm{cu}}=\left(\alpha_{1} \sum_{i=1}^{L}\left|h_{\mathrm{sr}_{i}}\right|^{2}\right) \bar{\gamma}_{s}
$$

implying that the IECU is capable of obtaining $L$-order of diversity for detection of the symbol transmitted by the source.

Let us express the symbol detected by the IECU as $\hat{x}$. Then, $\hat{x}$ is sent back to the $L$ relays without error, as the transmission from the IECU to relays is assumed ideal. Finally, every relay transmits $\hat{x}$ to the destination with the aid of the TEGC-assisted transmitter preprocessing, which can be described in (4) by setting $x_{r_{i}}=\hat{x}$. Correspondingly, the final decision variable formed at the destination can be expressed as (5) with $x_{r_{i}}=\hat{x}$, i.e.,

$$
y_{d}=\sum_{i=1}^{L} \sqrt{\frac{\alpha_{2}}{L}}\left|h_{r_{i} d}\right| \hat{x}+n_{d}
$$

\subsection{CRP}

In the ICRP, it is assumed that information exchange among the relays does not consume energy, which is obviously impractical. In this subsection, we consider the CRP scheme, which takes into account of the energy spent for information exchange among the relays and, hence, is a practical relay processing scheme. By comparing the achievable performance of the CRP with that of the ICRP considered in Section 3.2, we will realize that using ideal assumptions for cooperation may greatly overestimate the achievable performance of cooperative wireless systems.

When the CRP scheme is employed, the $L$ relays first transmit the signals received from the source to the IECU in the principles of AF relay. At the IECU, signals received from the $L$ relays are detected in the MRC principles. Then, the IECU broadcasts the detected symbol back to the $L$ relays. Finally, the relays use the DRP scheme to transmit their detected symbols to the destination.

In more detail, the CRP is operated as follows: Once the $L$ relays obtain the observations of $\boldsymbol{y}_{r}$, as shown in (1), each of the relays, say relay $i$, firstly normalizes its observation, forming $s_{r_{i}}=y_{r_{i}} / \sqrt{\left|h_{\mathrm{sr}_{i}}\right|^{2}+2 \sigma^{2}}(i=1,2, \ldots, L)$. Then, the $L$ relays forward their normalized observations to the
IECU with the aid of DS-CDMA. Correspondingly, the observations obtained by the IECU can be represented as

$$
\begin{aligned}
\boldsymbol{y}_{\mathrm{cu}}=\sqrt{\frac{\alpha_{\mathrm{ma}}}{L}} \boldsymbol{C} \boldsymbol{A}_{\mathrm{ma}} \boldsymbol{s}_{r}+\boldsymbol{n}_{\mathrm{cu}} & =\sqrt{\frac{\alpha_{\mathrm{ma}}}{L}} \boldsymbol{H}_{\mathrm{ma}} \boldsymbol{s}_{r}+\boldsymbol{n}_{\mathrm{cu}} \\
& =\sqrt{\frac{\alpha_{\mathrm{ma}}}{L}} \boldsymbol{C} \boldsymbol{A}_{\mathrm{ma}} \boldsymbol{G}_{r} \boldsymbol{y}_{r}+\boldsymbol{n}_{\mathrm{cu}}
\end{aligned}
$$

where, by definition, $y_{\mathrm{cu}}$ is a length- $N$ vector, when a spreading factor of $N$ is used by the DS-CDMA, $\boldsymbol{s}_{r}=\left[s_{r_{1}}, s_{r_{2}}, \ldots, s_{r_{L}}\right]^{T}, \boldsymbol{H}_{\mathrm{ma}}=\boldsymbol{C} \boldsymbol{A}_{\mathrm{ma}}$, where $\boldsymbol{C}=$ $\left[\boldsymbol{c}_{1}, \boldsymbol{c}_{2}, \ldots, \boldsymbol{c}_{L}\right]$ with $\left\|\boldsymbol{c}_{i}\right\|^{2}=1$ is a $(N \times L)$ matrix containing the $L$ spreading sequences assigned to the $L$ relays and $\boldsymbol{A}_{\text {ma }}=\operatorname{diag}\left\{a_{1}, a_{2}, \ldots, a_{L}\right\}$ with $a_{i}$ representing the fading gain of the $i$ th channel. We assume that $\left|a_{i}\right|$ obeys the Nakagami- $m$ distribution with the PDF expressed as $f_{\left|a_{i}\right|}(r)$ in the form of (3) and the parameters $m_{\mathrm{ma}_{i}}$ and $\Omega_{\mathrm{ma}_{i}}$. In (10), $\boldsymbol{G}_{r}=\operatorname{diag}\left\{g_{1}, g_{2}, \ldots, g_{L}\right\}$, where $g_{i}=1 / \sqrt{\left|h_{\mathrm{sr}_{i}}\right|^{2}+2 \sigma^{2}}(i=1,2, \ldots, L)$. Finally, in (10), $\boldsymbol{n}_{\mathrm{cu}}=\left[n_{\mathrm{cu}_{1}}, n_{\mathrm{cu}_{2}}, \ldots, n_{\mathrm{cu}_{N}}\right]^{T}$, the elements of which obey the complex Gaussian distribution with zero mean and a variance of $2 \sigma_{\mathrm{cu}}^{2}$ with $\sigma_{\mathrm{cu}}^{2}=1 /\left(2 \bar{\gamma}_{s} \beta_{1}\right)$, where $\beta_{1}$ is relate to the noise variance of the MA channels. Note that, it can be shown that the average SNR of each of the DS-CDMA channels is $\gamma_{\mathrm{ma}}=\alpha_{\mathrm{ma}} \bar{\gamma}_{s} \beta_{1} / L$.

When substituting (1) into (10), we can obtain an explicit expression relating to the symbol transmitted by the source, which is

$$
\begin{aligned}
\boldsymbol{y}_{\mathrm{cu}} & =\sqrt{\frac{\alpha_{1} \alpha_{\mathrm{ma}}}{L}} \boldsymbol{H}_{\mathrm{ma}} \boldsymbol{G}_{r} \boldsymbol{h}_{\mathrm{sr}} x+\sqrt{\frac{\alpha_{\mathrm{ma}}}{L}} \boldsymbol{H}_{\mathrm{ma}} \boldsymbol{G}_{r} \boldsymbol{n}_{r}+\boldsymbol{n}_{\mathrm{cu}} \\
& =\sqrt{\frac{\alpha_{1} \alpha_{\mathrm{ma}}}{L}} \boldsymbol{h}_{T} \boldsymbol{x}+\boldsymbol{n}_{T}
\end{aligned}
$$

where, for simplicity, we expressed $\boldsymbol{h}_{T}=\boldsymbol{H}_{\mathrm{ma}} \boldsymbol{G}_{r} \boldsymbol{h}_{\mathrm{sr}}$, which is a length- $N$ vector and can be viewed as the equivalent source to IECU channel matrix. Similarly, in (11), the length- $N$ noise vector $\boldsymbol{n}_{T}=\sqrt{\frac{\alpha_{\mathrm{ma}}}{L}} \boldsymbol{H}_{\mathrm{ma}} \boldsymbol{G}_{r} \boldsymbol{n}_{r}+\boldsymbol{n}_{\mathrm{cu}}$ contains the noise conflicted at both the relays and the IECU.

When the IECU employs $\boldsymbol{h}_{T}$, which can be directly estimated at the IECU without requiring to know $\boldsymbol{H}_{\mathrm{ma}}, \boldsymbol{G}_{r}$ and $\boldsymbol{h}_{\text {sr }}$ separately, it derives the decision variable in the MRC principle as

$$
\begin{aligned}
z_{\mathrm{cu}} & =\boldsymbol{h}_{T}^{H} \boldsymbol{y}_{\mathrm{cu}} \\
& =\sqrt{\frac{\alpha_{1} \alpha_{\mathrm{ma}}}{L}}\left|\boldsymbol{h}_{T}\right|^{2} x+\boldsymbol{h}_{T}^{H} \boldsymbol{n}_{T}
\end{aligned}
$$

Based on $z_{\mathrm{cu}}$, the IECU detects the symbol transmitted by the source, which is expressed as $\hat{x}$.

Then, the IECU broadcasts the detected symbol $\hat{x}$ to the $L$ relays, and the $i$ th relay obtains the observation

$$
\hat{y}_{r_{i}}=\sqrt{\alpha_{\mathrm{bc}}} h_{\mathrm{bc}_{i}} \hat{x}+\hat{n}_{r_{i}}, \quad i=1,2, \ldots, L
$$


where $\hat{n}_{r_{i}}$ is the Gaussian noise added on the $i$ th $\mathrm{BC}$ channel, which has zero mean and a variance of $\sigma_{\hat{r}}^{2}=$ $1 /\left(2 \bar{\gamma}_{s} \beta_{2}\right)$ per dimension, here $\beta_{2}$ is related to the noise variance of the $\mathrm{BC}$ channels. In (13), the channel is assumed to experience Nakagami- $m$ fading with $\left|h_{\mathrm{bc}_{i}}\right|$ obeying the Nakagami- $m$ distribution of (3) associated with the parameters $m_{\mathrm{bc}_{i}}$ and $\Omega_{\mathrm{bc}_{i}}$. From (13), we can know that the average SNR of a $\mathrm{BC}$ channel is $\gamma_{\mathrm{bc}}=$ $\alpha_{\mathrm{bc}} \bar{\gamma}_{s} \beta_{2}$.

Based on $\left\{\hat{y}_{r_{i}}\right\}$, the relays can make their decisions about the symbol transmitted by the IECU. Let the symbols detected by the $L$ relays be expressed as $\left\{x_{r_{i}}\right\}$. They are forwarded respectively by the $L$ relays to the destination, after the TEGC-assisted preprocessing, as detailed in section 3.1. Note again that, since the TEGC-assisted preprocessing invokes $L$ relays for transmitting signals to the destination, a diversity order of $L$ can be achieved by the CRP scheme.

Note that, if we let $m_{\mathrm{ma}_{i}} \rightarrow \infty$ and $m_{\mathrm{bc}_{i}} \rightarrow \infty$ in the PDFs for the MA and BC channels, then, the MA and BC channels are reduced to the AWGN channels [28].

\section{Analysis of bit error rate}

In this section, we analyze the BER of the THCL system employing the relay processing schemes considered in section 3, when BPSK baseband modulation is assumed. Our analysis is based on the assumptions that the $\mathrm{S}-\mathrm{R}$ channels, the MA channels and BC channels for information exchange, and the R-D channels experience independent fading. Specifically, the S-R channels experience the independent and identically distributed (iid) Nakagami$m$ fading, the same occurs with the $\mathrm{MA} / \mathrm{BC}$ channels and the R-D channels. However, the fading parameters characterizing the S-R channels, MA/BC channels and the R-D channels may be different. Additionally, we consider the special cases, where the S-R and R-D channels experience Nakagami- $m$ fading, while the MA/BC channels are AWGN channels, in order to demonstrate that the energy spent for relay cooperation cannot be ignored even in this over-optimistic communication environments.

Before considering the specific scheme, we first note that the average BER of the BPSK communicating over flat Nakagami- $m$ fading channels can be formulated as $[29,30]$

$$
\begin{aligned}
P_{b}\left(m, \gamma_{c}\right)= & \sqrt{\frac{\gamma_{c}}{\gamma_{c}+m}} \frac{\left(1+\gamma_{c} / m\right)^{-m} \Gamma(m+1 / 2)}{2 \sqrt{\pi} \Gamma(m+1)} \\
& \times{ }_{2} F_{1}\left(1, m+1 / 2 ; m+1 ; \frac{m}{m+\gamma_{c}}\right)
\end{aligned}
$$

where $\gamma_{c}$ represents the average SNR and ${ }_{2} F_{1}(a, b ; c ; z)$ is the hypergeometric function defined as [31] ${ }_{2} F_{1}(a, b ; c ; z)=\sum_{k=0}^{\infty} \frac{(a)_{k}(b)_{k} z^{k}}{(c)_{k} k !}$ with $(a)_{k}=a(a+1) \ldots$ $(a+k-1)$ and $(a)_{0}=1$.
Note that, there are a range of special forms for (14), which can be found in [28-30].

\subsection{Bit error rate of DRP}

From the principles of the THCL, as described in section 2, we can know that, when the DRP is employed, the errors of the $L \mathrm{~S}-\mathrm{R}$ channels occur independently. Hence, the average BER can be expressed as

$$
\bar{P}_{b}^{(\mathrm{DRP})}=\sum_{l=0}^{L}\left(\begin{array}{c}
L \\
l
\end{array}\right)\left(1-P_{b}^{(\mathrm{S}-\mathrm{R})}\right)^{l}\left(P_{b}^{(\mathrm{S}-\mathrm{R})}\right)^{q} P_{b}^{(\mathrm{TEGC})}(l)
$$

where $P_{b}^{(\mathrm{S}-\mathrm{R})}$ is the average BER of S-R channels and $P_{b}^{(\mathrm{TEGC})}(l)$ is the average BER of the destination's detection on the condition that $l$ out of $L$ relays send the destination correct bits, while the other $q=L-l$ relays send the destination erroneous bits.

For the S-R transmission, the observations obtained by the relays are given in (1). Hence, when the $S-R$ channels are assumed Nakagami- $m$ fading channels associated with the parameters $\left(m_{\mathrm{sr}}, \Omega_{\mathrm{sr}}\right)$, the average BER of $P_{b}^{(\mathrm{S}-\mathrm{R})}$ in (15) is then given by

$$
P_{b}^{(\mathrm{S}-\mathrm{R})}=P_{b}\left(m=m_{\mathrm{sr}}, \gamma_{c}=\alpha_{1} \Omega_{\mathrm{sr}} \bar{\gamma}_{s}\right)
$$

where $P_{b}\left(m, \gamma_{c}\right)$ is given by $(14)$.

Considering the transmission from the $L$ relays to the destination, if there are $l$ relays sending the destination correct bits and $q=L-l$ relays sending the destination erroneous bits, the decision variable formed by the destination is given by (6), i.e. $y_{d}^{(l, q)}=\sqrt{\frac{\alpha_{2}}{L}} h_{l, q} x+n_{d}$ where $h_{l, q}=h_{\sum l}-h_{\sum q}$ with $h_{\sum l}=\sum_{i \in l\{1, \ldots, L\}}\left|h_{r_{i} d}\right|$ and $h_{\sum q}=\sum_{j \hat{\epsilon}_{q}\{1, \ldots, L\}}\left|h_{r_{j} d}\right|$. Hence, in order to derive the BER expression, we need to derive the PDF of $h_{l, q}$. Below, we derive this PDF by first introducing the Nakagami approximation for the PDFs of $h_{\sum l}$ and $h_{\sum q}$. Two types of Nakagami approximation approaches are proposed, which are the modified Nakagami theoretical approximation (Nakagami-TAp) and the Nakagami statistical approximation (Nakagami-SAp).

In the context of the Nakagami-TAp, first, according to [32], when the $l$ components in $h_{\sum l}$ are independent and obey the same Nakagami- $m$ distribution with the parameters $m_{0}$ and $\Omega_{0}, h_{\sum l}$ can be approximated as a Nakagami- $m$ distributed random variable with the $\operatorname{PDF} f_{h_{\sum l}}\left(y \mid m_{l}, \Omega_{l}\right)$ in the form of (3) associated with the parameters

$$
m_{l}=l \times m_{0}, \Omega_{l}=l^{2} \times \Omega_{0}
$$

However, as the results in [32] show, the above approximation may be very inaccurate.

Based on our careful studies and numerous simulation verifications, we find that the $\operatorname{PDF} f_{h_{\sum l}}\left(y \mid m_{l}, \Omega_{l}\right)$ can be 
slightly modified to make it very accurate, yielding the modified Nakagami-TAp, which is expressed as

$$
f_{h_{\sum l}}^{\mathrm{Mod}}\left(y \mid m_{l}, \Omega_{l}\right)=f_{h_{\sum l}}\left(y \mid m_{l}, \kappa \Omega_{l}\right)
$$

where $\kappa$ is a coefficient which is dependent on the distribution of the components in $h_{\sum l}$ and the value of $l$. For instance, when $\Omega_{0}=1$, a range of values for $\kappa$ have been found, which are summarized in Table 1. From the table, we see that $\kappa<1$ is always the case. This implies that the approximation using the parameters in (17) overestimates $\Omega_{l}$.

When the Nakagami-SAp is employed, we approximate $h_{\sum l}$ as a Nakagami- $m$ distributed random variable with its PDF $f_{h l}\left(y \mid m_{l}, \Omega_{l}\right)$ expressed in the form of (3), whose parameters $m_{l}$ and $\Omega_{l}$ are obtained by simulations. Note that, the Nakagami- $m$ PDF is not very sensitive to the values of $m$ and $\Omega$, especially, when these values are relatively large. For example, the PDFs of $f_{\left|h_{i j}\right|}(y \mid m, \Omega)$ do not have any noticeable differences, when $\Omega \pm 0.01 \Omega$ and $m \pm 0.01 m$ are applied. Hence, it is usually sufficient for us to derive $m$ and $\Omega$ based on about $10^{3}$ to $10^{4}$ realizations of $h_{i j}$. Hence, the time spent for using the Nakagami-SAp to obtain BER results can be significantly less than that required by using direct simulations. When using direct simulations, we know that at least $10^{7}$ (independent) realizations are required for a BER of about $10^{-5}$, in order to generate sufficient accuracy.

Note furthermore that, the Nakagami-SAp is very general and robust, as it does not require the details of the component distributions. However, if the parameters of the component distributions are known, the parameters for the Nakagami-SAp are only required to be generated once by simulation, which can then be repeatedly used for performance evaluation. For instance, we can make a table, like Table 2 , showing the parameters, $\left(m_{L}, \Omega_{L}\right)$, for the resultant Nakagami- $m$ distributions of $\sum_{l=1}^{L}\left|h_{l}\right|$, when $L$ takes different values and the components, $\left\{\left|h_{l}\right|\right\}$, obey the iid Nakagami- $m$ distributions with different values for the parameters $\left(m_{0}, \Omega_{0}\right)$. The values of $\left(m_{L}, \Omega_{L}\right)$ shown in the table were generated based on $10^{4}$ realizations. Some of these values were used for generating the

Table 1 Parameters $\kappa$ for PDF of $h_{\sum l} l$ obtained by Nakagami-TAp, where component distributions have parameter $\Omega_{0}=1$

\begin{tabular}{ccccc}
\hline $\boldsymbol{l}$ & \multicolumn{5}{c}{} \\
\cline { 2 - 5 } & $\mathbf{1 . 0}$ & $\mathbf{1 . 5}$ & $\mathbf{3 . 0}$ & $\mathbf{4 . 0}$ \\
\hline 2 & 0.842 & 0.879 & 0.914 & 0.924 \\
3 & 0.825 & 0.867 & 0.914 & 0.929 \\
4 & 0.812 & 0.862 & 0.915 & 0.930 \\
5 & 0.807 & 0.859 & 0.918 & 0.931 \\
6 & 0.803 & 0.857 & 0.919 & 0.933 \\
\hline
\end{tabular}

performance results shown in Section 5. Explicitly, they generate very accurate approximation. Furthermore, this table can be repeatedly used and possibly for different applications.

Having obtained the $\operatorname{PDF} f_{h_{\sum l}}\left(y \mid m_{l}, \Omega_{l}\right)$ of $h_{\sum l}$ and also the PDF $f_{h_{\sum q}}\left(y \mid m_{q}, \Omega_{q}\right)$ of $h_{\sum q}$, the PDF of $h_{l, q}$ in (6) can be derived, as shown in the Appendix, and can be expressed as

$$
\begin{aligned}
f_{h_{l, q}}(x)= & \frac{4 m_{l}^{m_{l}} m_{q}^{m_{q}}}{\Omega_{l}^{m_{l}} \Omega_{q}^{m_{q}} \Gamma\left(m_{l}\right) \Gamma\left(m_{q}\right)} \exp \left(\frac{m_{q}^{2}-m_{q} \Omega_{q} \eta^{2}}{\Omega_{q}^{2} \eta^{2}} x^{2}\right) \\
& \times \int_{u \eta-\frac{m_{q} x}{\Omega_{q} \eta}}^{\infty} \frac{1}{\eta}\left(\frac{s}{\eta}+\frac{m_{q} x}{\Omega_{q} \eta^{2}}\right)^{2 m_{l}-1} \\
& \times\left(\frac{s}{\eta}+\frac{m_{q} x}{\Omega_{q} \eta^{2}}-x\right)^{2 m_{q}-1} e^{-s^{2}} d s
\end{aligned}
$$

in the general cases. In (19), $u=\max \{0, x\}$ and $\eta=$ $\sqrt{\frac{m_{l}}{\Omega_{l}}+\frac{m_{q}}{\Omega_{q}}}$. However, if $\left(2 m_{l}-1\right)$ and $\left(2 m_{q}-1\right)$ are integers, the above formula can be expressed as

$$
\begin{aligned}
f_{h_{l, q}}(x)= & \frac{2 m_{l}^{m_{l}} m_{q}^{m_{q}}}{\Omega_{l}^{m_{l}} \Omega_{q}^{m_{q}} \Gamma\left(m_{l}\right) \Gamma\left(m_{q}\right)} \exp \left(\frac{-m_{l} m_{q} x^{2}}{m_{l} \Omega_{q}+m_{q} \Omega_{l}}\right) \\
& \times \sum_{l_{1}=0}^{2 m_{l}-1} \sum_{q_{1}=0}^{2 m_{q}-1}\left(\begin{array}{c}
2 m_{l}-1 \\
l_{1}
\end{array}\right)\left(\begin{array}{c}
2 m_{q}-1 \\
q_{1}
\end{array}\right) \\
& \times\left(\frac{m_{q}}{\Omega_{q} \eta^{2}}\right)^{2 m_{l}-1-l_{1}}\left(\frac{m_{q}}{\Omega_{q} \eta^{2}}-1\right)^{2 m_{q}-1-q_{1}} \times\left(\frac{1}{\eta}\right)^{l_{1}+q_{1}+1} \\
& \times \Gamma\left(\frac{l_{1}+q_{1}+1}{2},\left(u \eta-\frac{m_{q} x}{\Omega_{q} \eta}\right)^{2}\right) x^{2 m_{l}+2 m_{q}-l_{1}-q_{1}-2}
\end{aligned}
$$

where $\Gamma(a, x)$ represents the incomplete gamma function given by (40).

When given $h_{l, q}$, the BER of the R-D transmission employing the DRP can be derived based on (6), which can be expressed as

$$
P_{b}^{\text {(TEGC) }}\left(l \mid h_{l, q}\right)=Q\left(h_{l, q} \sqrt{\frac{2 \alpha_{2} \bar{\gamma}_{s}}{L}}\right), 0 \leq l \leq L
$$

where $Q(x)$ represents the Gaussian $Q$ function [33], which is defined as $Q(x)=\frac{1}{\sqrt{2 \pi}} \int_{x}^{\infty} e^{-t^{2} / 2} d t$. Alternatively, when $x>0, Q(x)$ can also be expressed as $Q(x)=$ $\frac{1}{\pi} \int_{0}^{\pi / 2} \exp \left(-\frac{x^{2}}{2 \sin ^{2} \theta}\right) d \theta$ [34]. Correspondingly, the average BER of the R-D transmission can be expressed as

$$
P_{b}^{(\mathrm{TEGC})}(l)= \begin{cases}\int_{-\infty}^{\infty} Q\left(x \sqrt{\frac{2 \alpha_{2} \bar{\gamma}_{s}}{L}}\right) f_{h_{l, q}}(x) d x, & \text { if } 0<l<L \\ \int_{0}^{\infty} Q\left(x \sqrt{\frac{2 \alpha_{2} \bar{\gamma}_{s}}{L}}\right) f_{h_{\Sigma L}}(x) d x, & \text { if } l=L \\ 1-\int_{0}^{\infty} Q\left(x \sqrt{\frac{2 \alpha_{2} \bar{\gamma}_{s}}{L}}\right) f_{h_{\Sigma L}}(x) d x, & \text { if } l=0\end{cases}
$$


Table 2 Parameters $\left(m_{L}, \Omega_{L}\right)$ for PDF of $h_{\sum L}=\sum_{l=1}^{L}\left|h_{l}\right|$ obtained by Nakagami-SAp

\begin{tabular}{|c|c|c|c|c|c|c|c|}
\hline \multirow{2}{*}{$L$} & \multirow{2}{*}{$\Omega_{0}$} & \multicolumn{6}{|c|}{$m_{0}$} \\
\hline & & 0.5 & 1 & 1.5 & 2 & 2.5 & 3 \\
\hline \multirow[t]{4}{*}{2} & 0.125 & $0.929,0.424$ & $1.908,0.443$ & $2.929,0.46$ & $3,948,0.469$ & $4.918,0.474$ & $5.869,0.477$ \\
\hline & 0.25 & $0.929,0.808$ & $1.908,0.886$ & $2.929,0.919$ & $3,948,0.938$ & $4.918,0.948$ & $5.869,0.958$ \\
\hline & 0.5 & $0.929,1.617$ & $1.908,1.771$ & $2.929,1.838$ & $3,948,1.875$ & $4.918,1.895$ & $5.869,1.904$ \\
\hline & 1 & $0.929,3.234$ & $1.908,3.542$ & $2.929,3.676$ & $3,948,3.751$ & $4.918,3.791$ & $5.869,3.819$ \\
\hline \multirow[t]{4}{*}{3} & 0.125 & $1.36,0.843$ & $2.851,0.957$ & $4.314,1.007$ & $5.872,1.038$ & $7.303,1.052$ & $8.809,1.061$ \\
\hline & 0.25 & $1.36,1.687$ & $2.851,1.913$ & $4.314,2.015$ & $5.872,2.077$ & $7.303,2.105$ & $8.809,2.122$ \\
\hline & 0.5 & $1.36,3.373$ & $2.851,3.826$ & $4.314,4.029$ & $5.872,4.154$ & $7.303,4.21$ & $8.809,4.244$ \\
\hline & 1 & $1.36,6.746$ & $2.851,7.652$ & $4.314,8.059$ & $5.872,8.307$ & $7.303,8.419$ & $8.809,8.487$ \\
\hline \multirow[t]{4}{*}{4} & 0.125 & $1.776,1.443$ & $3.758,1.67$ & $5.731,1.77$ & $7.737,1.828$ & $9.693,1.858$ & $11.842,1.871$ \\
\hline & 0.25 & $1.776,2.885$ & $3.758,3.34$ & $5.731,3.541$ & $7.737,3.656$ & $9.693,3.715$ & $11.842,3.742$ \\
\hline & 0.5 & $1.776,5.77$ & $3.758,6.68$ & $5.731,7.082$ & $7.737,7.312$ & $9.693,7.431$ & $11.842,7.485$ \\
\hline & 1 & $1.776,11.54$ & $3.758,13.36$ & $5.731,14.163$ & $7.737,14.624$ & $9.693,14.861$ & $11.842,14.97$ \\
\hline \multirow[t]{4}{*}{5} & 0.125 & $2.226,2.207$ & $4.656,2.58$ & $7.082,2.746$ & $9.696,2.835$ & $12.12,2.891$ & $14.66,2.916$ \\
\hline & 0.25 & $2.226,4.414$ & $4.656,5.161$ & $7.082,5.491$ & $9.696,5.67$ & $12.12,5.782$ & $14.66,5.831$ \\
\hline & 0.5 & $2.226,8.829$ & $4.656,10.322$ & $7.082,10.982$ & $9.696,11.34$ & $12.12,11.564$ & $14.66,11.662$ \\
\hline & 1 & $2.226,17.658$ & $4.656,20.645$ & $7.082,21.964$ & $9.696,22.68$ & $12.12,23.128$ & $14.66,23.324$ \\
\hline \multirow[t]{4}{*}{6} & 0.125 & $2.647,3.13$ & $5.636,3.688$ & $8.538,3.932$ & $11.6,4.067$ & $14.511,4.149$ & $17.685,4.189$ \\
\hline & 0.25 & $2.647,6.26$ & $5.636,7.376$ & $8.538,7.864$ & $11.6,8.314$ & $14.511,8.299$ & $17.685,8.378$ \\
\hline & 0.5 & $2.647,12.519$ & $5.636,14.752$ & $8.538,15.728$ & $11.6,16.268$ & $14.511,16.597$ & $17.685,16.756$ \\
\hline & 1 & $2.647,25.038$ & $5.636,29.505$ & $8.538,31.456$ & $11.6,32.536$ & $14.511,33.195$ & $17.685,33.512$ \\
\hline
\end{tabular}

$L$ components are iid Nakagami- $m$ random variables with parameters $\left(m_{0}, \Omega_{0}\right)$.

Consequently, the average BER of $P_{b}^{(\mathrm{TEGC})}(l)$ can be evaluated from (22) by invoking (19) or (20). Specifically, when $\left(2 m_{l}-1\right)$ and $\left(2 m_{q}-1\right)$ are integers, it can be shown that

$$
\begin{aligned}
P_{b}^{(\mathrm{TEGC})}(l)= & \frac{m_{l}^{m_{l}} m_{q}^{m_{q}}}{\Omega_{l}^{m_{l}} \Omega_{q}^{m_{q}} \Gamma\left(m_{l}\right) \Gamma\left(m_{q}\right)} \sum_{l_{1}=0}^{2 m_{l}-1} \sum_{q_{1}=0}^{2 m_{q}-1}\left(\begin{array}{c}
2 m_{l}-1 \\
l_{1}
\end{array}\right)\left(\begin{array}{c}
2 m_{q}-1 \\
q_{1}
\end{array}\right) \\
& \times\left(\frac{m_{q}}{\Omega_{q} \eta^{2}}\right)^{2 m_{l}-1-l_{1}}\left(\frac{m_{q}}{\Omega_{q} \eta^{2}}-1\right)^{2 m_{q}-1-q_{1}}\left(\frac{1}{\eta}\right)^{l_{1}+q_{1}+1} \\
& \times \int_{-\infty}^{\infty} Q\left(x \sqrt{2 \alpha_{2} \bar{\gamma}_{s} / L}\right) \exp \left(\frac{-m_{l} m_{q} x^{2}}{m_{l} \Omega_{q}+m_{q} \Omega_{l}}\right) \\
& \times \Gamma\left(\frac{l_{1}+q_{1}+1}{2},\left(u \eta-\frac{m_{q} x}{\Omega_{q} \eta}\right)^{2}\right) x^{2 m_{l}+2 m_{q}-l_{1}-q_{1}-2} d x
\end{aligned}
$$

when $0<l<L$, and when $l=L$,

$$
P_{b}^{(\mathrm{TEGC})}(L)=P_{b}\left(m=m_{L}, \gamma_{c}=\alpha_{2} \Omega_{L} \bar{\gamma}_{s} / L\right)
$$

where $P_{b}\left(m, \gamma_{c}\right)$ is given by (14).

Finally, the average BER of the THCL system employing the DRP can be evaluated from (15) with the aid of (23) and (24).

\subsection{Bit error rate of ICRP}

When the ICRP scheme is considered, as shown in Section 3.2, the bits transmitted by the $L$ relays to the destination are just the bit detected by the IECU. Hence, the average BER of the THCL system using the ICRP scheme can be written as

$\bar{P}_{b}^{(\mathrm{ICRP})}=P_{b}^{(\mathrm{IECU})}\left(1-P_{b}^{(\mathrm{TEGC})}\right)+\left(1-P_{b}^{(\mathrm{IECU})}\right) P_{b}^{(\mathrm{TEGC})}$

In (25), the first (second) term at the right-hand side denotes the probability that the detection at the IECU is incorrect (correct) while the detection at the destination is correct (incorrect).

First, in (25), $P_{b}^{(\mathrm{TEGC})}$ is the average BER of detection at the destination, which has been analyzed in section 4.1. In the ICRP scenario, all the $L$ bits to be transmitted by the relays are the same. Hence, we have $P_{b}^{\text {(TEGC) }}=$ $P_{b}^{(\mathrm{TEGC})}(L)$, which is given by (24).

Second, in (25), $P_{b}^{(\mathrm{IECU})}$ is the BER of the detection at the IECU. When the MRC is employed, the decision variable is given by (7) with the average SNR given by (8). The BER of this problem has been analyzed in many references, such as, in [28-30]. It can be shown that, when the 
$L$ S-R channels experience independent Nakagami- $m$ fading with the same PDF determined by the parameters $m_{\mathrm{sr}}$ and $\Omega_{\mathrm{sr}}$, the average BER of the IECU's detection can be expressed as [28-30]

$$
\begin{aligned}
P_{b}^{(\mathrm{IECU})}= & \sqrt{\frac{\alpha_{1} \Omega_{\mathrm{sr}} \bar{\gamma}_{s}}{\alpha_{1} \Omega_{\mathrm{sr}} \bar{\gamma}_{s}+m_{\mathrm{sr}}}} \frac{\left(1+\alpha_{1} \Omega_{\mathrm{sr}} \bar{\gamma}_{s} / m_{\mathrm{sr}}\right)^{-m_{\mathrm{sr}} L} \Gamma\left(m_{\mathrm{sr}} L+1 / 2\right)}{2 \sqrt{\pi} \Gamma\left(m_{\mathrm{sr}} L+1\right)} \\
& \times{ }_{2} F_{1}\left(1, m_{\mathrm{sr}} L+1 / 2 ; m_{\mathrm{sr}} L+1 ; \frac{m_{\mathrm{sr}}}{m_{\mathrm{sr}}+\alpha_{1} \Omega_{\mathrm{sr}} \bar{\gamma}_{s}}\right)
\end{aligned}
$$

in the general cases. Furthermore, when $m_{\mathrm{sr}} L$ is a positive integer, (26) can be reduced to

$$
P_{b}^{(\mathrm{IECU})}=\left[\frac{1-\mu}{2}\right]^{m_{\mathrm{sr}} L} \sum_{v=0}^{m_{\mathrm{sr}} L-1}\left(\begin{array}{c}
m_{\mathrm{sr}} L-1+v \\
v
\end{array}\right)\left[\frac{1+\mu}{2}\right]^{v}
$$

where $\mu=\sqrt{\alpha_{1} \Omega_{\mathrm{sr}} \bar{\gamma}_{s} /\left(\alpha_{1} \Omega_{\mathrm{sr}} \bar{\gamma}_{s}+m_{\mathrm{sr}}\right)}$ by definition.

\subsection{Bit error rate of CRP}

Finally, when the CRP scheme is considered, errors may occur over the S-R channels, MA/BC channels for information exchange and the R-D channels. Let the BER after the IECU's detection be $P_{b}^{(\mathrm{IECU})}$ and the BER of the BC channels be $P_{b}^{(\mathrm{BC})}$. Then, $P_{b}^{(\mathrm{S}-\mathrm{R})}$ in (15), which represents the BER of the bits to be sent to the destination, can be expressed as

$$
\begin{aligned}
P_{b}^{(\mathrm{S}-\mathrm{R})} & =P_{b}^{(\mathrm{IECU})}\left(1-P_{b}^{(\mathrm{BC})}\right)+\left(1-P_{b}^{(\mathrm{IECU})}\right) P_{b}^{(\mathrm{BC})} \\
& =P_{b}^{(\mathrm{IECU})}+P_{b}^{(\mathrm{BC})}-2 P_{b}^{(\mathrm{IECU})} P_{b}^{(\mathrm{BC})}
\end{aligned}
$$

Substituting (28) into (15), we can express the average BER of the THCL system employing the CRP as

$$
\begin{aligned}
\bar{P}_{b}^{(\mathrm{CRP})}= & \sum_{l=0}^{L}\left(\begin{array}{c}
L \\
l
\end{array}\right)\left(1-P_{b}^{(\mathrm{IECU})}-P_{b}^{(\mathrm{BC})}+2 P_{b}^{(\mathrm{IECU})} P_{b}^{(\mathrm{BC})}\right)^{l} \\
& \times\left(P_{b}^{(\mathrm{IECU})}+P_{b}^{(\mathrm{BC})}-2 P_{b}^{(\mathrm{IECU})} P_{b}^{(\mathrm{BC})}\right)^{L-l} P_{b}^{(\mathrm{TEGC})}(l)
\end{aligned}
$$

where $P_{b}^{(\text {TEGC })}(l)$ is the same as that analyzed in Section 4.1. The BER $P_{b}^{(\mathrm{BC})}$ of the BC channels can be readily obtained. The decision variables formed by the $L$ relays for the $\mathrm{BC}$ channels are given in (13). Based on this equation, the average BER of the $\mathrm{BC}$ channels is

$$
P_{b}^{(\mathrm{BC})}=P_{b}\left(m=m_{\mathrm{bc}}, \gamma_{c}=\alpha_{\mathrm{bc}} \beta_{2} \Omega_{\mathrm{bc}} \bar{\gamma}_{s}\right)
$$

when the $\mathrm{BC}$ channels are assumed to experience independent Nakagami- $m$ fading with the parameters $m_{\mathrm{bc}}$ and $\Omega_{\mathrm{bc}}$. When the BC channels are AWGN channels, we simply have $P_{b}^{(\mathrm{BC})}=Q\left(\sqrt{2 \alpha_{\mathrm{bc}} \beta_{2} \bar{\gamma}_{s} / L}\right)$.

In (29), $P_{b}^{(\text {IECU) }}$ is the BER of the detection at the IECU using MRC. In this case, the decision variable formed by the IECU is expressed as (12), based on which the instantaneous SNR can be expressed as

$$
\gamma_{\mathrm{cu}}=\frac{\mathrm{E}^{2}\left[z_{\mathrm{cu}}\right]}{\operatorname{Var}\left[z_{\mathrm{cu}}\right]}=\frac{\alpha_{1} \alpha_{\mathrm{ma}} \bar{\gamma}_{s}}{L} \frac{\left(\boldsymbol{h}_{T}^{H} \boldsymbol{h}_{T}\right)^{2}}{\frac{\alpha_{\mathrm{ma}}}{L} \sum_{i=1}^{L}\left|h_{D_{i}}\right|^{2}+\sum_{j=1}^{N}\left|h_{T_{j}}\right|^{2}}
$$

where we defined $\boldsymbol{h}_{D}^{T}=\left[h_{D_{1}}, h_{D_{2}}, \ldots, h_{D_{L}}\right]=\boldsymbol{h}_{T}^{H} \boldsymbol{H}_{\mathrm{ma}} \boldsymbol{G}_{r}$. However, it is extremely hard to derive the exact PDF of $\gamma_{\mathrm{cu}}$ from (31), due to the forwarded noise by the relays, as seen in (11). Consequently, we are incapable of deriving the exact average BER of $P_{b}^{(\text {IECU) }}$. In this paper, we propose the Gamma approximation (Gamma-Ap) for obtaining the PDF of $\gamma_{\mathrm{cu}}$.

Note that, in performance analysis, the Gaussian approximation (Gaussian-Ap) is typically employed. However, for some scenarios, such as for the PDF of (31), where the concerned variables are always positive, the Gamma-Ap has the advantages over the GaussianAp. First, the Gamma distribution [29], which can be obtained by the squares of Nakagami- $m$ distributed variables, is defined in $[0, \infty)$, while the Gaussian distribution is defined in $(-\infty, \infty)$. Second, for applying the GaussianAp, usually a high number of component variables is required, so that their sum yields a symmetric distribution. By contrast, the Gamma-Ap does not impose this constraint, and can be applied for the sum of any number of component variables. Furthermore, as the number of components increases, the resultant Gamma distribution appears the Gaussian-like shape, but, in the range $[0, \infty)$. Hence, the Gamma-Ap (also including the Nakagami approximation, as they belong to the same family) represents a versatile approximation approach, which may find applications for a lot of problems in practice, including a lot of performance analysis problems in wireless communications.

Specifically, for the current case, let us rewrite (31) as

$$
\gamma_{\mathrm{cu}}=\frac{\alpha_{1} \alpha_{\mathrm{ma}} \bar{\gamma}_{s}}{L} \zeta_{\mathrm{cu}}
$$

where

$$
\zeta_{\mathrm{cu}}=\frac{\left(\boldsymbol{h}_{T}^{H} \boldsymbol{h}_{T}\right)^{2}}{\frac{\alpha_{\mathrm{ma}}}{L} \sum_{i=1}^{L}\left|h_{D_{i}}\right|^{2}+\sum_{j=1}^{N}\left|h_{T_{j}}\right|^{2}}
$$

Then, with the Gamma-Ap, we can approximate $\zeta_{\mathrm{cu}}$ as a Gamma distributed random variable with the PDF [29]

$$
f_{\zeta_{\mathrm{cu}}}(y)=\left(\frac{m_{\mathrm{cu}}}{\Omega_{\mathrm{cu}}}\right)^{m_{\mathrm{cu}}} \frac{y^{m_{\mathrm{cu}}-1}}{\Gamma\left(m_{\mathrm{cu}}\right)} \exp \left(-\frac{m_{\mathrm{cu}} y}{\Omega_{\mathrm{cu}}}\right)
$$

where $\Omega_{\mathrm{cu}}=E\left[\zeta_{\mathrm{cu}}\right]$, and $m_{\mathrm{cu}}=\Omega_{\mathrm{cu}}^{2} / E\left[\left(\zeta_{\mathrm{cu}}-\Omega_{\mathrm{cu}}\right)^{2}\right]$.

From (33), we can see that the parameters $m_{\mathrm{cu}}$ and $\Omega_{\mathrm{cu}}$ determining the PDF of $\zeta_{\mathrm{cu}}$ depend on the average SNR $\bar{\gamma}_{s}$ of the S-R channels through the matrix $\boldsymbol{G}_{r}$ in $\boldsymbol{h}_{D}$, the fading of the $L \mathrm{~S}-\mathrm{R}$ channels, the fading of the MA channels and the spreading factor $N$ of the DS-CDMA signaling. 
Hence, it may be extremely hard (if it is not impossible) to derive the parameters $m_{\mathrm{cu}}$ and $\Omega_{\mathrm{cu}}$ by mathematical analysis. However, they can be readily found by simulations based on about $10^{4}$ realizations. For instance, in Table 3, a range of cases are considered, where the spreading factor $N$ is 16 , the $L \mathrm{~S}-\mathrm{R}$ channels experience the same Nakagami- $m$ fading with the parameters $m_{\mathrm{sr}}$ and $\Omega_{\mathrm{sr}}=1$, and the MA channels also experience the same Nakagami$m$ fading with the parameters $m_{\mathrm{ma}}$ and $\Omega_{\mathrm{ma}}=1$. As our results in Section 5, the Gamma-Ap in general yields very accurate approximation.

With the aid of the Gamma-Ap for finding the PDF of $\zeta_{\text {cu }}$, which is (34), we can now easily obtain the average BER of the detection at the IECU, which can be expressed as

$$
P_{b}^{(\mathrm{IECU})}=P_{b}\left(m=m_{\mathrm{cu}}, \gamma_{c}=\alpha_{1} \alpha_{\mathrm{ma}} \Omega_{\mathrm{cu}} \bar{\gamma}_{s} / L\right)
$$

Furthermore, the average of the THCL system employing the CRP scheme can be evaluated from (29) associated with (22) (or (23) and (24)), (30) and (35).

\section{Performance results}

In this section, we demonstrate a range of performance results for characterizing the achievable performance of THCL systems with the various relay processing schemes considered. Both numerical results evaluated from the formulas derived in the previous sections and simulation results are provided. Note that, for obtaining the results, we assume that all channels of the first and second hops experience independent fading. The MA/BC channels are either Gaussian channels for illustrating the best cases or iid fading channels. When the CRP is employed, we assume that the parameters $\beta_{1}$ and $\beta_{2}$ take a value of 10 , which results in that the average SNR of the MA/BC channels is typically $10 \mathrm{~dB}$ higher than that of the S-R and R-D channels. Furthermore, for the DS-CDMA used for

Table 3 Parameters for the Gamma PDF $f_{\zeta_{\mathrm{cu}}}(\zeta)$ obtained based on the Gamma-Ap

\begin{tabular}{cccccc}
\hline $\boldsymbol{L}$ & $\overline{\boldsymbol{\gamma}}_{\boldsymbol{s}}(\mathbf{d B})$ & $\boldsymbol{m}_{\mathbf{s r}}$ & $\boldsymbol{m}_{\mathbf{m a}}$ & $\boldsymbol{m}_{\mathbf{c u}}$ & $\boldsymbol{\Omega}_{\mathbf{c u}}$ \\
\hline 4 & 1 & 1.0 & 1.0 & 2.573 & 18.121 \\
& 4 & 2.0 & 3.0 & 7.347 & 27.007 \\
& 9 & 1.0 & 4.0 & 5.332 & 29.775 \\
& 14 & 3.0 & 1.5 & 7.730 & 33.445 \\
& 20 & 2.5 & 1.5 & 6.401 & 33.150 \\
6 & 3 & 1.5 & 1.0 & 4.872 & 37.363 \\
& 7 & 1.0 & 1.0 & 4.506 & 43.158 \\
& 13 & 3.0 & 3.0 & 13.320 & 64.612 \\
& 18 & 1.0 & 4.0 & 5.189 & 49.028 \\
& 22 & 1.5 & 3.0 & 6.199 & 55.084 \\
\hline
\end{tabular}

the MA transmission, the spreading codes are assumed random sequences with a spreading factor $N=16$.

In Figures 2 and 3, we compare the approximate BER of the DRP-assisted THCL with the corresponding BER obtained by simulations, when the S-R and R-D channels are assumed iid Nakagami- $\boldsymbol{m}$ fading channels. Furthermore, Figures 2 and 3 illustrate the impacts of the number of relays and the fading parameter $m$ on the achievable BER performance. Note that, the approximate BER was evaluated based on (15), when either the NakagamiTAp or the Nakagami-SAp was employed. From Figure 2, we can observe that there is a slight deviation between the approximate BER and the simulated BER, when the Nakagami-TAp is applied. However, the difference becomes smaller as the Nakagami- $m$ fading becomes less severe, i.e., as $m$ increases. The difference also becomes smaller, as the number of relays increases. Nevertheless, for all the scenarios considered, the approximate BER and simulated BER are close to each other. When the Nakagami-SAp is employed, as shown in Figure 3, the approximate BER and the simulated BER always agree with each other. Therefore, we are confident that both the Nakagami-TAp and Nakagami-SAp are highly effective, while the Nakagami-SAp is more accurate than the Nakagami-TAp.

Additionally, as shown in Figures 2 and 3, the BER performance improves as the fading becomes less severe. It also improves as the number of relays increases, owing to the increased spatial diversity.

The BER performance of the THCL employing ICRP is shown in Figure 4, when assuming that both the S-R and R-D channels experience iid Nakagami- $m$ fading. The theoretical BER was evaluated based on the Nakagami-TAp. Again, from the results of Figure 4, we can observe that the approximate BER of all the considered cases closely matches the corresponding BER obtained by simulations. In comparison with the results shown in Figure 2 or Figure 3, we can see that remarkable SNR gain may be attained, when the ICRP is employed by an IECU instead of using the DRP. Note that, this performance gain achieved by the ICRP-assisted THCL is mainly due to the cooperative detection of the first hop without considering the energy consumption. This impractical cooperation detection generates the symbols having much higher reliability than that detected by the relays operated under the practical DRP scheme.

When practical non-ideal cooperation is assumed, Figure 5 shows the BER performance of the THCL employing CRP, when $1 / 3$ of the total transmission power is allocated for S-R, MA/BC and R-D transmission, respectively. In our simulations, we assumed that all the S-R and R-D channels are iid Nakagami- $m$ fading channels with a fading parameter $m_{\mathrm{sr}}$, while the MA/BC 


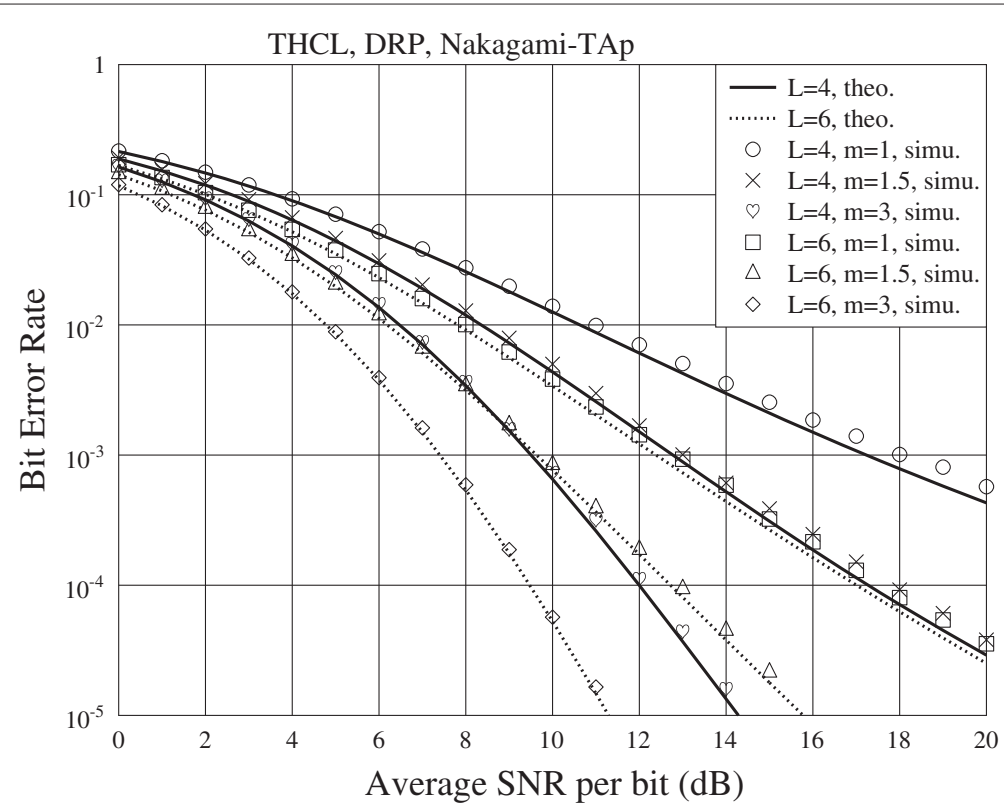

Figure 2 BER of DRP-assisted THCL employing BPSK baseband modulation, power allocation factors $\alpha_{1}=\alpha_{2}=0.5$, and Nakagami-TAp approach.

channels are iid Nakagami- $m$ fading channels with a fading parameter $m_{\mathrm{ma}}$. In this figure, the approximate BER was obtained based on the Gamma-Ap for the detection at the IECU and the Nakagami-TAp for the detection at the destination. From the results shown in the figure, we can have the following observations. First, the approximate BER agrees with the corresponding BER obtained by simulations in the relatively low SNR region, but there appears some deviation in the high SNR region. Second, the approximate BER becomes more accurate, as channel fading becomes less severe. Third, the approximate BER also becomes more accurate as the first and/or second hops becomes more reliable. Additionally, when comparing the results shown in Figure 5 with those shown in Figure 4, we are implied that the practically achievable BER performance of the THCL should be much worse

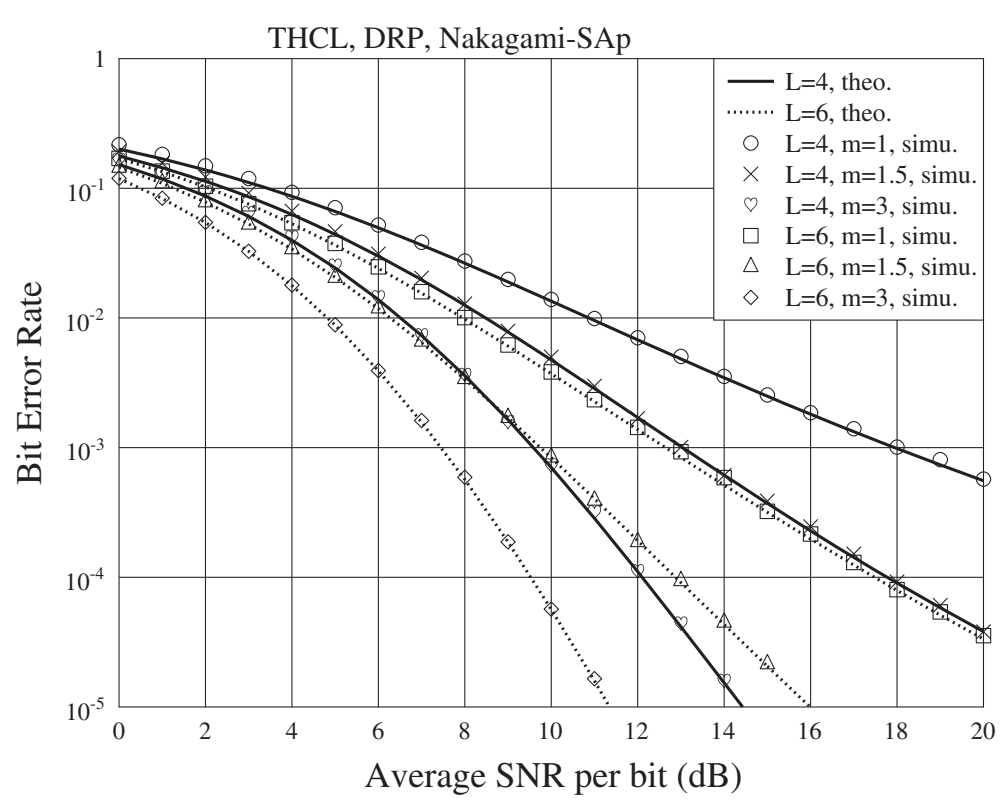

Figure 3 BER of DRP-assisted THCL employing BPSK baseband modulation, power allocation factors $\alpha_{1}=\alpha_{2}=0.5$, and Nakagami-SAp approach. 


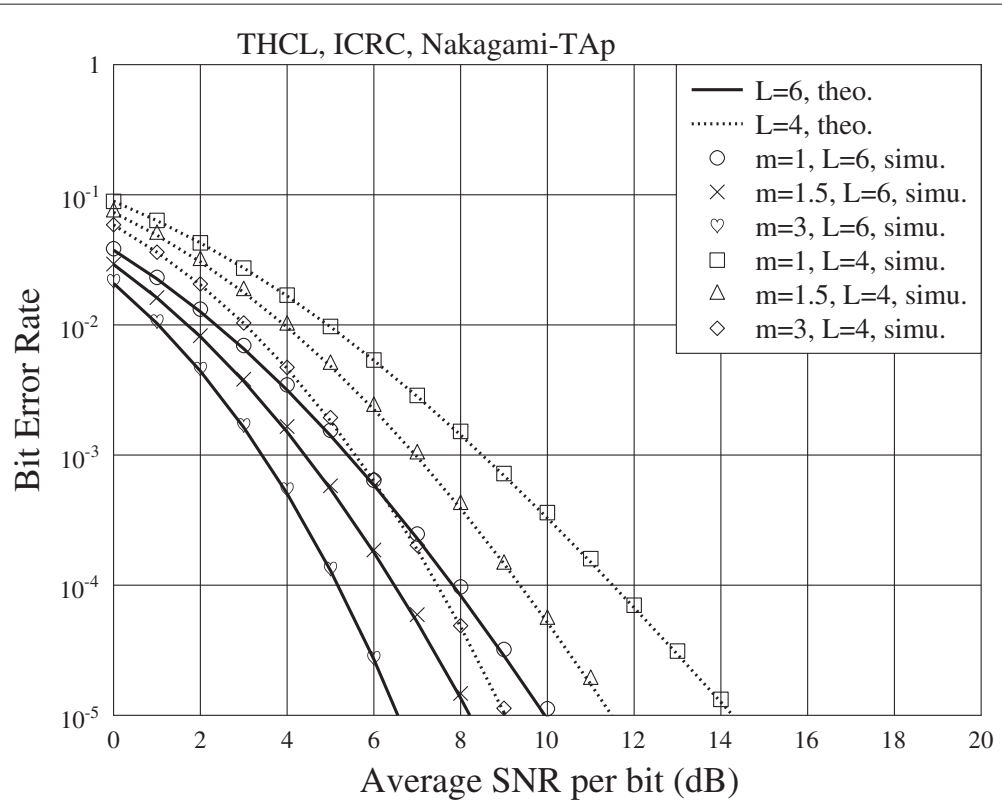

Figure 4 BER of ICRP-assisted THCL employing BPSK baseband modulation and power allocation factors $\alpha_{1}=\alpha_{2}=0.5$.

than that predicted from the ICRP, as the relays require energy for cooperation. Note that, as shown at the beginning of this section, the $\mathrm{MA} / \mathrm{BC}$ channels are in fact assumed more reliable than the S-R and R-D channels. This issues will be further discussed later associated with the other figures.

In Figure 6, we demonstrate the impact of the reliability of the S-R and R-D channels on the achievable
BER performance of the THCLs. In our simulations, we assumed that the S-R and R-D channels are Nakagami$m$ fading channels with a fading parameter $m_{\mathrm{sr}}$ and $m_{\mathrm{rd}}$, respectively, while the $\mathrm{MA} / \mathrm{BC}$ channels are iid Nakagami$m$ fading channels with a fading parameter $m_{\mathrm{ma}}$. The values for these parameters were $m_{\mathrm{ma}}=m_{\mathrm{bc}}=1$, while $m_{\mathrm{sr}}=1$ and $m_{\mathrm{rd}}=2$ or $m_{\mathrm{sr}}=2$ and $m_{\mathrm{rd}}=1$. Since a larger value for the fading parameter means that the

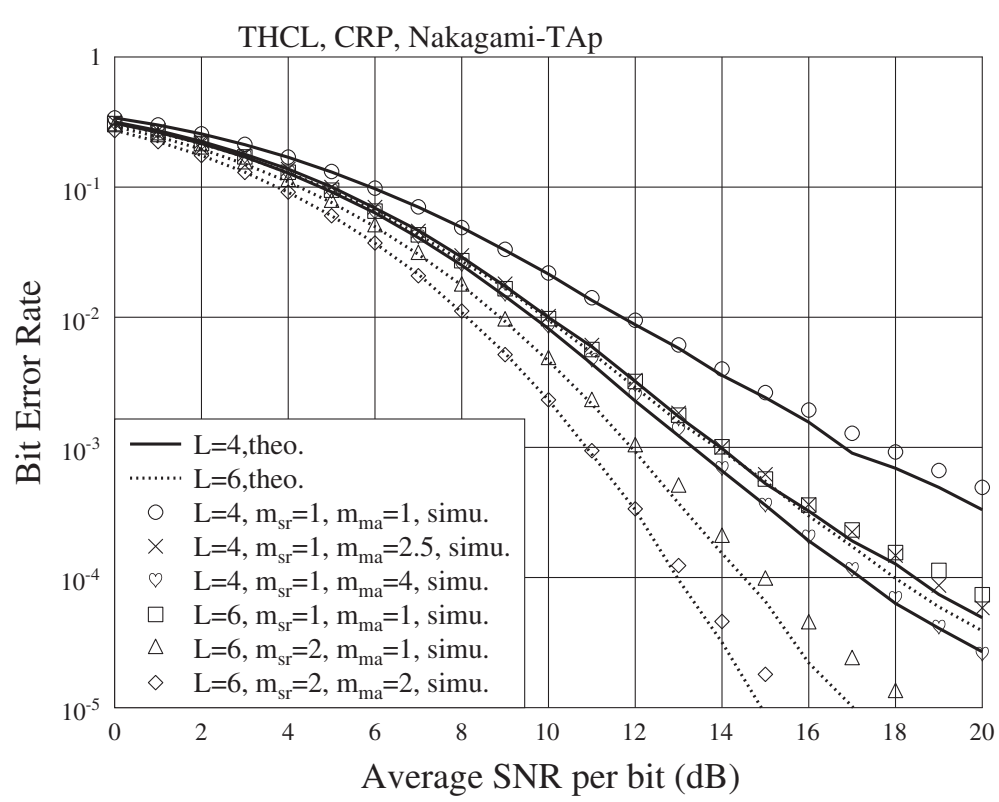

Figure 5 BER of the CRP-assisted THCL employing BPSK baseband modulation and power allocation factors $\alpha_{1}=\alpha_{2}=\alpha_{r}=1 / 3$. All the S-R and R-D channels are assumed iid Nakagami- $m$ fading channels with a fading parameter of $m_{\mathrm{sr}}$ while the MA/BC channels, with a fading parameter of $m_{m a}$. 


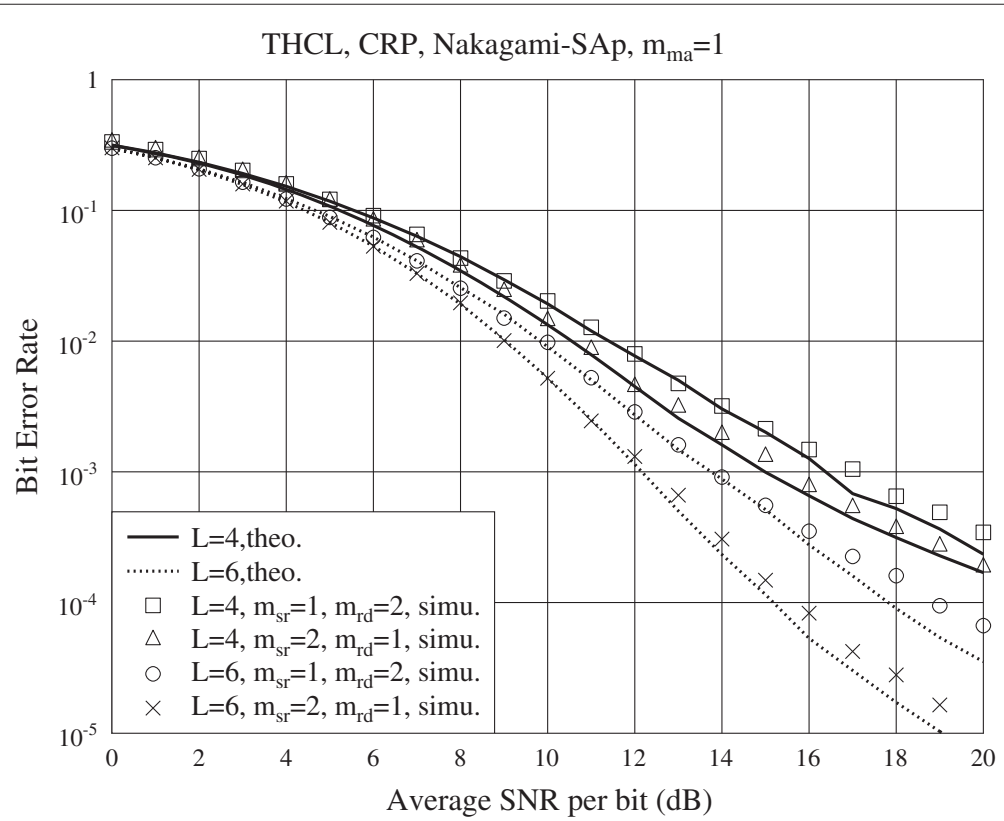

Figure 6 BER of CRP-assisted THCL employing BPSK baseband modulation and power allocation factors $\alpha_{1}=\alpha_{2}=\alpha_{r}=1 / 3$. The S-R and R-D channels are assumed Nakagami- $m$ fading channels with a fading parameter of $m_{s r}$ and $m_{r d}$, respectively while the MA/BC channels, with a fading parameter of $m_{\mathrm{ma}}$.

corresponding channel is more reliable, from Figure 6, we observe that having reliable $S-R$ channels is more important than having reliable R-D channels, in order to improve the BER performance of the THCL. This observation becomes more explicit, when the number of relays is increased from $L=4$ to $L=6$.

In Figures 7 and 8, we demonstrate the impact of power allocation on the achievable BER performance of the THCL employing the CRP, when both the S-R and the
R-D experience iid flat Rayleigh fading. For the MA/BC channels, AWGN channels are assumed for Figure 7, in order to illustrate that even in this case, the performance achievable by the practical CRP may be much worse than that achieved by the ICRP, implying that the ideal assumptions applied are highly impractical. By contrast, flat Rayleigh fading channels are assumed for Figure 8. In these figures, the 'optimum point' indicates the optimum power allocation and the corresponding

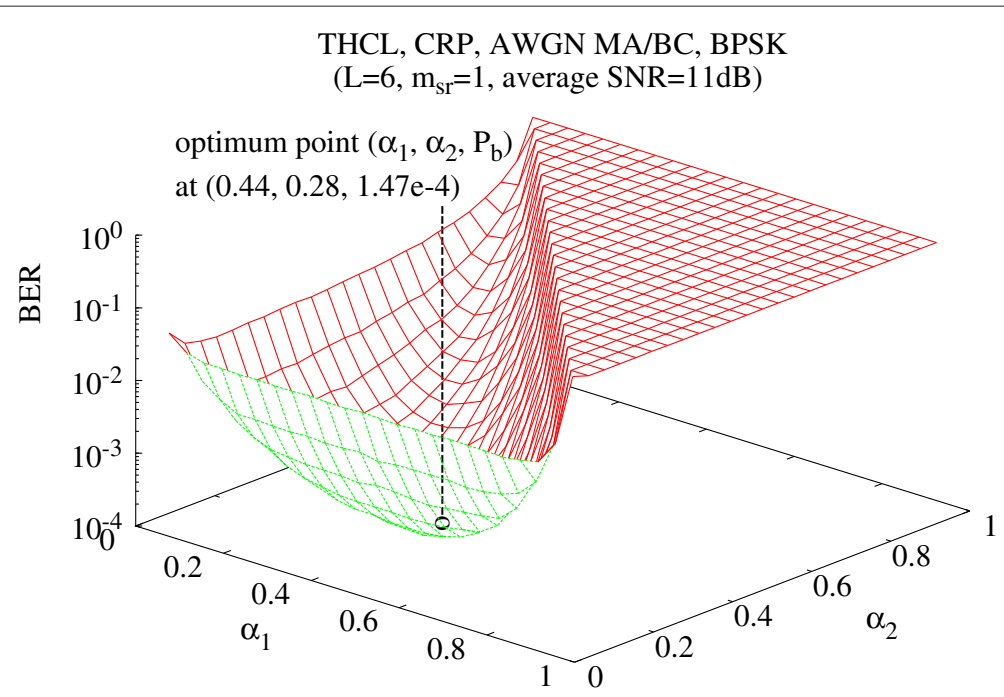

Figure 7 Effect of power allocation factors on BER performance of CRP-assisted THCL employing BPSK baseband modulation. Both the first and second hop channels experience flat iid Rayleigh fading, while the MA/BC channels are AWGN channels. 
THCL, CRP, Fading MA/BC, BPSK

$\left(\mathrm{L}=6, \mathrm{~m}_{\mathrm{sr}}=1, \mathrm{~m}_{\mathrm{ma}}=1\right.$, average $\left.\mathrm{SNR}=11 \mathrm{~dB}\right)$

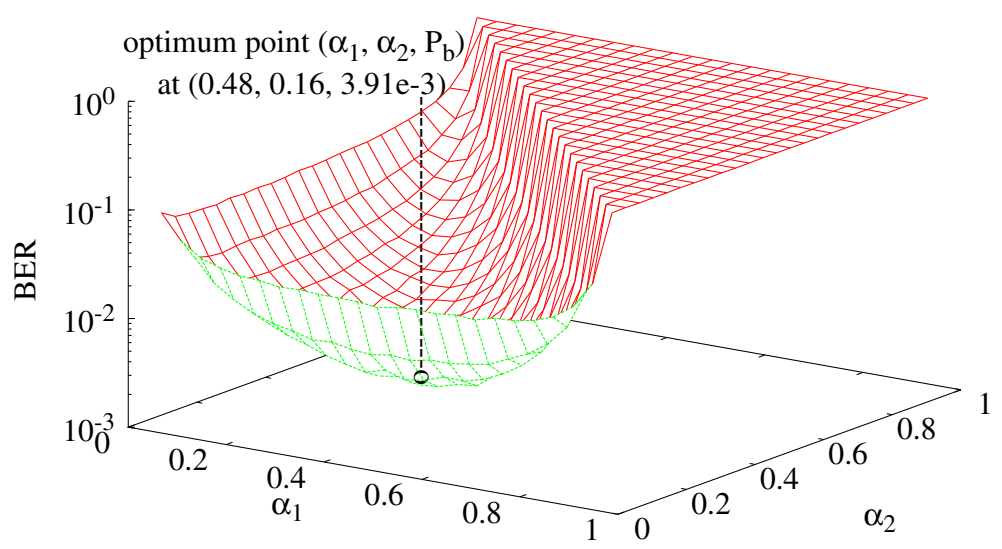

Figure 8 Effect of power allocation factors on BER performance of CRP-assisted THCL employing BPSK baseband modulation. All the S-R, $M A / B C$ and R-D channels experience flat iid Rayleigh fading.

lowest BER achievable. As seen in Figure 7 for the AWGN $\mathrm{MA} / \mathrm{BC}$ scenario, the optimum power allocation is $\alpha_{1}=$ $0.44, \alpha_{2}=0.28, \alpha_{r}=0.28$. Hence, the main portion of power is allocated to the first hop to improve the reliability of the first hop to a sufficient level. By contrast, when the MA/BC channels are also Rayleigh fading channels, as seen in Figure 8, the power allocation for the THCL system to achieve the best BER performance is $\alpha_{1}=0.48, \alpha_{2}=0.16, \alpha_{r}=0.36$. More portion of the total power is required for information exchange among the relays, compared with the AWGN MA/BC case. From both the figures, we are informed that a big portion of energy is required for implementing cooperation among relays. Hence, in wireless networks, using cooperative relays is in fact highly challenging. Not only is a substantial amount of energy is required for cooperation, the accompanied increase of complexity may be substantial as well. This is because, first, extra channel estimation is required. Second, power allocation will become more difficult, as four hops need to be considered in a network employing cooperative relays, instead of two hops in a network using distributed relays.

Additionally, we may compare the best BER achieved in Figure 7, which assumed AWGN MA/BC channels, with Figure 4. In Figure 4, the curve corresponding to the parameters of $m=1, L=6$ shows that the BER at $11 \mathrm{~dB}$ is well below $10^{-5}$. This BER is much lower than the best BER of $1.47 \times 10^{-4}$ shown in Figure 7 . From this comparison, we are implied that the BER predicted by applying ideal assumptions is far overoptimistic.

Finally, in Figures 9 and 10, we compare the BER performance achieved by the DRP, ICRP, and CRP, when applying the suboptimal power allocation scheme. Under this power allocation scheme, the optimum power allocation at a certain SNR is used for similar SNRs, instead of finding the optimum power allocation for every simulated SNR. For the channels, in Figure 9, we assumed that the $\mathrm{S}-\mathrm{R}, \mathrm{MA} / \mathrm{BC}$, and R-D channels are all flat Rayleigh fading channels. By contrast, in Figure 10, we assumed that the S-R and R-D channels are flat Rayleigh fading channels, while MA/BC channels are flat Nakagami- $m$ fading channels associated with a fading parameter $m_{\mathrm{ma}}=3$. Explicitly, for any a case and a given SNR, the BER achieved by the ICRP is much lower than that attained by the DRP and the CRP, as information exchange among relays in the ICRP does not consume energy. When comparing the DRP with the CRP, first, in the cases of $m_{\mathrm{ma}}=3$, as shown in Figure 10, the CRP outperforms the DRP, when the SNR is sufficiently high. However, if the SNR is low (such as, lower than $8 \mathrm{~dB}$ ), the DRP outperforms the CRP. Second, in the cases of $m_{\mathrm{ma}}=1$, as seen in Figure 9, the DRP outperforms the CRP within the considered SNR region, which becomes more explicit when the SNR decreases. The reason for this is that relay cooperation becomes less reliable, when either the MA/BC channels become worse or the noise power increases (i.e., average SNR reduces). As the CRP requires extra channel estimation and centralized detection, its achievable BER performance will be sensitive to the channel estimations accuracy, in addition to the added complexity. When taking into account of all the above, we conclude that the DRP possibly constitutes a desirable and practical relay processing scheme. It has relatively low complexity for implementation, achieves diversity from the TEGC and yields less processing delay owing to no information exchange among relays. 


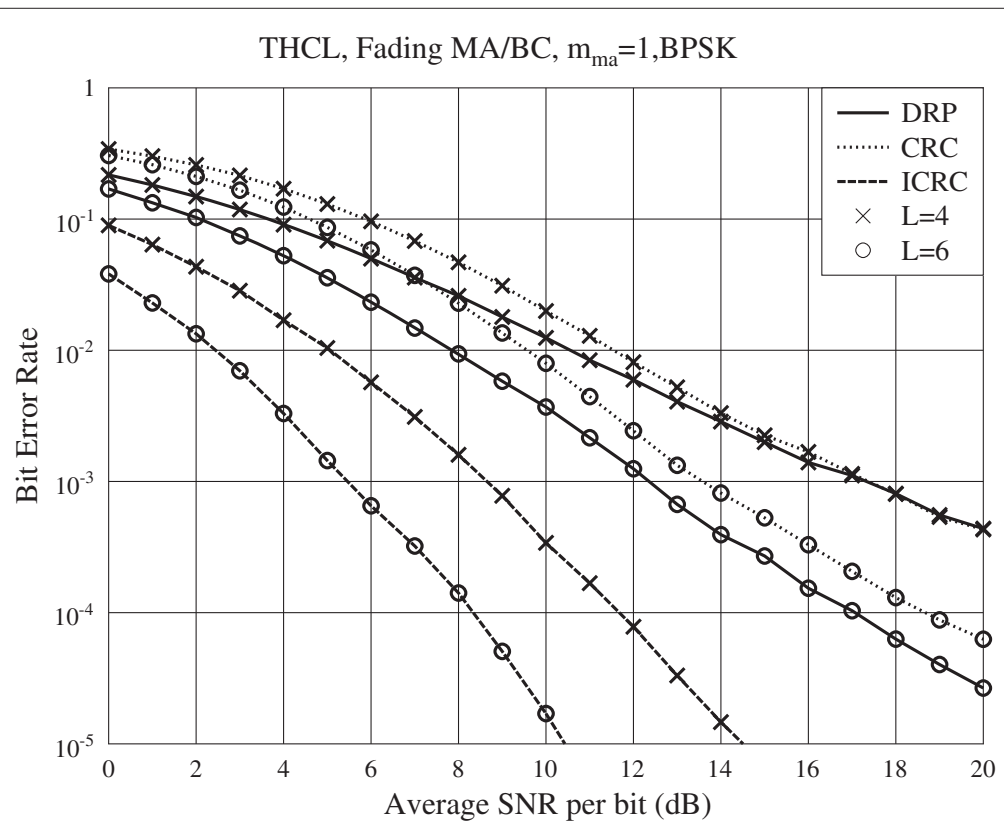

Figure 9 Comparison of BER performance of THCL systems employing DRP, ICRP, and CRP. The S-R, MA/BC, and R-D channels are flat Rayleigh fading channels.

\section{Conclusions}

In this paper, we have studied the BER performance of THCL systems with various relay processing schemes, including the DRP, ICRP, and CRP. As the BER of the TEGC-assisted detection at the destination is hard to analyze, two approximation approaches have been proposed, which are the Nakagami-TAp and Nakagami-SAp.
Our performance studies show that both the approximation approaches can be confidently used for predicting the BER of the schemes generating EGC-type decision variables, although the Nakagami-SAp may yield more accurate results than the Nakagami-TAp. For evaluation of the BER at the IECU, the Gamma-Ap is proposed for finding the distribution of the related SINR. From

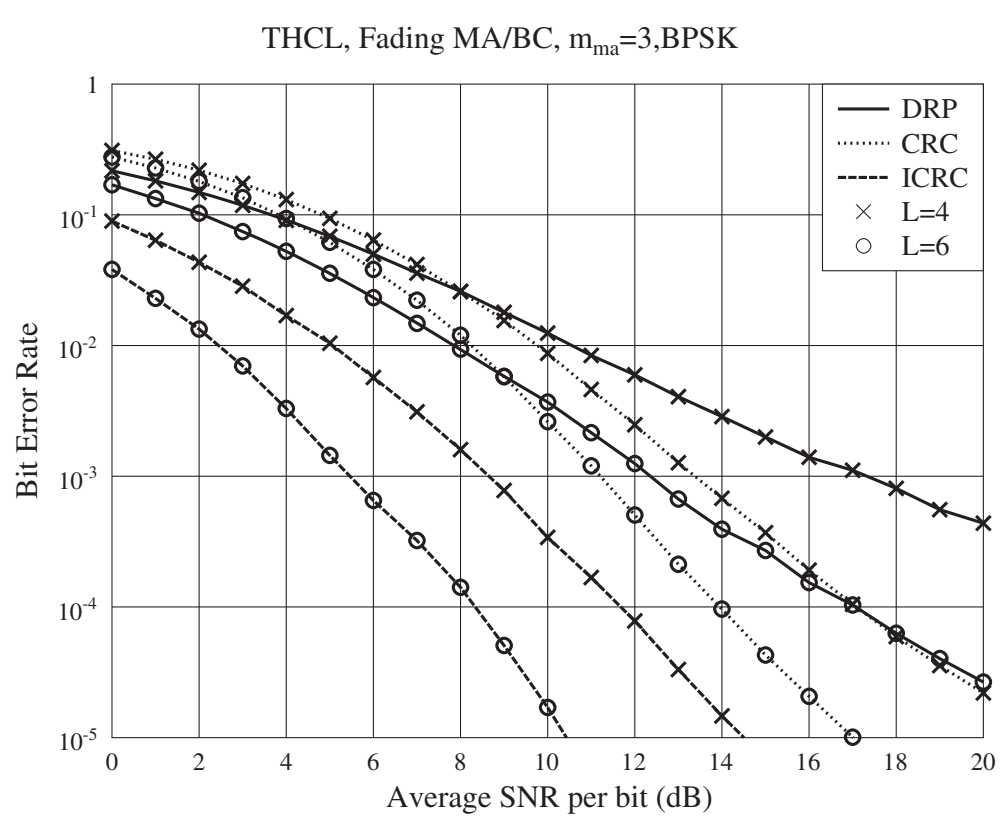

Figure 10 Comparison of BER performance of THCL systems employing DRP, ICRP, and CRP. Both the S-R and R-D channels are flat Rayleigh fading channels and the MA/BC channels are flat Nakagami- $m$ fading channels associated with a fading parameter of $m_{m a}=3$. 
our studies, we can realize that these approximation approaches, especially, the Gamma-Ap, are highly general, which may find applications in performance analysis of a wide range of communication systems. Finally, the BER performance of the various relay processing schemes has been demonstrated and compared. From the performance results, we can conclude that, without considering the cost for relay cooperation, the ICRP always outperforms the DRP. However, in the practical scenarios where relay cooperation consumes energy, bandwidth, and implementation complexity, we find that the DRP often outperforms the CRP even in terms of the BER performance. Therefore, in relay communications, the performance predicted under the assumption of ideal relay cooperation may be too optimistic and, hence, unachievable in practice. Owing to its low complexity for implementation, in practice, the DRP constitutes a highly desirable relay processing scheme, especially, in the communication environments where a few, such as $L \geq 4$, of relays are available.

\section{Appendix}

Given that $h_{l, q}=h_{\sum l}-h_{\sum q}$, as shown in [35], we have

$$
f_{h_{l, q}}(x)=\int_{u}^{\infty} f_{h_{\sum l}}(y) f_{h_{\sum q}}(y-x) d y
$$

where $u=\max \{0, x\}, f_{h_{\sum l}}(y)=f\left(y \mid m_{l}, \Omega_{l}\right)$ and $f_{h_{\sum q}}(y)=$ $f\left(y \mid m_{q}, \Omega_{q}\right)$ are Nakagami- $m$ PDFs in the form of (3). Upon substituting these PDFs into (36) and after some arrangement, we obtain

$$
\begin{aligned}
f_{h_{b q}}(x)= & \frac{4 m_{l}^{m_{l}} m_{q}^{m_{q}}}{\Omega_{l}^{m_{l}} \Omega_{q}^{m_{q}} \Gamma\left(m_{l}\right) \Gamma\left(m_{q}\right)} \int_{u}^{\infty} y^{2 m_{l}-1}(y-x)^{2 m_{q}-1} \\
& \times \exp \left[-\frac{m_{l}}{\Omega_{l}} y^{2}-\frac{m_{q}}{\Omega_{q}}(y-x)^{2}\right] d y
\end{aligned}
$$

Let us apply the variable transform of $s=\eta y-\frac{m_{q} x}{\Omega_{q} \eta}$, where $\eta=\sqrt{\frac{m_{l}}{\Omega_{l}}+\frac{m_{q}}{\Omega_{q}}}$. Then, we can express the above formula as

$$
\begin{aligned}
f_{h_{l, q}}(x)= & \frac{4 m_{l}^{m_{l}} m_{q}^{m_{q}}}{\Omega_{l}^{m_{l}} \Omega_{q}^{m_{q}} \Gamma\left(m_{l}\right) \Gamma\left(m_{q}\right)} \exp \left(\frac{m_{q}^{2}-m_{q} \Omega_{q} \eta^{2}}{\Omega_{q}^{2} \eta^{2}} x^{2}\right) \\
& \times \int_{u \eta-\frac{m_{q} x}{\Omega_{q} \eta}}^{\infty} \frac{1}{\eta}\left(\frac{s}{\eta}+\frac{m_{q} x}{\Omega_{q} \eta^{2}}\right)^{2 m_{l}-1} \\
& \times\left(\frac{s}{\eta}+\frac{m_{q} x}{\Omega_{q} \eta^{2}}-x\right)^{2 m_{q}-1} e^{-s^{2}} d s
\end{aligned}
$$

In the general cases when $2 m_{l}-1$ and $2 m_{q}-1$ are arbitrary values, to the best of our knowledge, (38) cannot be further simplified. By contrast, when $2 m_{l}-1$ and $2 m_{q}-1$ are integers, it can be shown that, with the aid of the serial expansion, (38) can be expressed as

$$
\begin{aligned}
f_{h_{l, q}}(x)= & \frac{2 m_{l}^{m_{l}} m_{q}^{m_{q}}}{\Omega_{l}^{m_{l}} \Omega_{q}^{m_{q}} \Gamma\left(m_{l}\right) \Gamma\left(m_{q}\right)} \exp \left(\frac{-m_{l} m_{q} x^{2}}{m_{l} \Omega_{q}+m_{q} \Omega_{l}}\right) \\
& \times \sum_{l_{1}=0}^{2 m_{l}-12 m_{q}-1} \sum_{q_{1}=0}^{2 m_{l}-1}\left(_{1}\right)\left(\begin{array}{c}
2 m_{q}-1 \\
q_{1}
\end{array}\right)\left(\frac{m_{q}}{\Omega_{q} \eta^{2}}\right)^{2 m_{l}-1-l_{1}} \\
& \times\left(\frac{m_{q}}{\Omega_{q} \eta^{2}}-1\right)^{2 m_{q}-1-q_{1}} \times\left(\frac{1}{\eta}\right)^{l_{1}+q_{1}+1} \\
& \times \Gamma\left(\frac{l_{1}+q_{1}+1}{2},\left(u \eta-\frac{m_{q} x}{\Omega_{q} \eta}\right)^{2}\right) x^{2 m_{l}+2 m_{q}-l_{1}-q_{1}-2}
\end{aligned}
$$

where $\Gamma(a, x)$ represents the incomplete gamma function defined as [31]

$$
\Gamma(a, x)=\int_{x}^{\infty} t^{a-1} e^{-t} \mathrm{~d} t
$$

Note that, when $m_{l}$ and $m_{q}$ are relatively big, we may approximate $2 m_{l}-1$ and $2 m_{q}-1$ (sometimes even $m_{l}$ and $m_{q}$ ) to their nearest integers and still generate sufficient accuracy, as evidenced by the results shown in [12]. This is because the Nakagami- $m$ PDFs are not very sensitive to the value of $m$, when it is relatively big.

\section{Competing interests}

The authors declare that they have no competing interests.

\section{Acknowledgements}

This work was presented in part at the IEEE VTC2012 Spring, 6-9 May 2012, Yokohama, Japan.

Received: 14 November 2013 Accepted: 13 March 2014 Published: 4 April 2014

\section{References}

1. S Berger, M Kuhn, A Wittneben, T Unger, A Klein, Recent advances in amplify-and-forward two-hop relaying. IEEE Commun. Mag. 47(7), 50-56 (2009)

2. JN Laneman, DNC Tse, GW Wornell, Cooperative diversity in wireless networks: efficient protocols and outage behavior. IEEE Trans. Inform. Theory. 50(12), 3062-3080 (2004)

3. A Sendonaris, E Erkip, B Aazhang, User cooperation diversity. part I. system description. IEEE Trans. Commun. 51(11), 1927-1938 (2003)

4. A Sendonaris, E Erkip, B Aazhang, User cooperation diversity. part II. Implementation aspects and performance analysis. IEEE Trans. Commun. 51(11), 1939-1948 (2003)

5. AH Bastami, A Olfat, Optimal SNR-based selection relaying scheme in multi-relay cooperative networks with distributed space-time coding. IET Commun. 4(6), 619-630 (2010)

6. P Anghel, M Kaveh, Exact symbol error probability of a cooperative network in a Rayleigh-fading environment. IEEE Trans. Wireless Commun. 3(5), 1416-1421 (2004)

7. KJR Liu, AK Sadek, WF Su, A Kwasinski, Cooperative Communications and Networking. (Cambridge University Press, New York, 2009)

8. O Amin, S Ikki, M Uysal, On the performance analysis of multirelay cooperative diversity systems with channel estimation errors. IEEE Trans. Vehicular Technol. 60(5), 2050-2059 (2011)

9. C Dong, L-L Yang, L Hanzo, Performance analysis of multihop-diversityaided multihop links. IEEE Trans Vehicular Technol. 61(6), 2504-2516 (2012) 
10. P Anghel, M Kaveh, Exact symbol error probability of a cooperative network in a Rayleigh-fading environment. IEEE Trans Wireless Commun. 3(5), 1416-1421 (2004)

11. A Ribeiro, X Cai, G Giannakis, Symbol error probabilities for general cooperative links. IEEE Trans. Wireless Commun. 4(3), 1264-1273 (2005)

12. L-L Yang, $\mathrm{H}-\mathrm{H}$ Chen, Error probability of digital communications using relay diversity over Nakagami-m fading channels. IEEE Trans. Wireless Commun. 7(5), 1806-1811 (2008)

13. B Barua, $\mathrm{H} \mathrm{Ngo}, \mathrm{H}$ Shin, On the SEP of cooperative diversity with opportunistic relaying. IEEE Commun. Lett. 12(10), 727-729 (2008)

14. THimsoon, W Siriwongpairat, W Su, K Liu, Differential modulations for multinode cooperative communications. IEEE Trans. Signal Process. 56(7), 2941-2956 (2008)

15. J Boyer, D Falconer, $\mathrm{H}$ Yanikomeroglu, Multihop diversity in wireless relaying channels. IEEE Trans. Commun. 52(10), 1820-1830 (2004)

16. Y Zhao, R Adve, TJ Lim, Outage probability at arbitrary SNR with cooperative diversity. IEEE Commun. Lett. 9(8), 700-702 (2005)

17. HA Suraweera, PJ Smith, J Armstrong, Outage probability of cooperative relay networks in Nakagami-m fading channels. IEEE Commun. Lett. 10(12), 834-836 (2006)

18. A Bletsas, H Shin, M Win, Outage optimality of opportunistic amplify-and-forward relaying. IEEE Commun. Lett. 11(3), 261-263 (2007)

19. N Sagias, F Lazarakis, G Tombras, C Datsikas, Outage analysis of decode-and-forward relaying over Nakagami- $m$ fading channels. IEEE Signal Process. Lett. 15, 41-44 (2008)

20. G Kramer, M Gastpar, P Gupta, Cooperative strategies and capacity theorems for relay networks. IEEE Trans. Inform Theory. 51(9), 3037-3063 (2005)

21. A Host-Madsen, J Zhang, Capacity bounds and power allocation for wireless relay channels. IEEE Trans. Inform. Theory. 51(6), 2020-2040 (2005)

22. A Host-Madsen, Capacity bounds for cooperative diversity. IEEE Trans. Inform. Theory. 52(4), 1522-1544 (2006)

23. Y Chen, S Kishore, J Li, Wireless diversity through network coding. IEEE Wireless Commun. Network. Conf. (WCNC 2006). 3, 1681-1686 (2006)

24. J Laneman, GW Wornell, Distributed space-time-coded protocols for exploiting cooperative diversity in wireless networks. IEEE Trans. Inform. Theory. 49(10), 2415-2425 (2003)

25. J Shi, L-L Yang, Performance of two-hop communication links employing various relay processing schemes, in IEEE VTC 2012-Spring 6-9 May (IEEE, Piscataway, 2012)

26. D Gunduz, A Yener, A Goldsmith, H Poor, The multi-way relay channel, in IEEE International Symposium on Information Theory (ISIT 2009) (IEEE, Piscataway, 2009), pp. 339-343

27. J Shi, L-L Yang, Performance of multiway relay DS-CDMA systems over Nakagami-m fading channels, in IEEE 73rd Vehicular Technology Conference (VTC 2011-Spring) (IEEE, Piscataway, 2011), pp. 1-5

28. L-L Yang, L Hanzo, Performance of generalized multicarrier DS-CDMA, over Nakagami-m fading channels. IEEE Trans. Commun. 50(6), 956-966 (2002)

29. MK Simon, M-S Alouini, Digital Communication Over Fading Channels: A Unified Approach to Performance Analysis. (Wiley, New York, 2000)

30. L-L Yang, Multicarrier Communications. (Wiley, Chichester, 2009)

31. IS Gradshteyn, IM Ryzhik, Table of Integrals, Series, and Products. (Academic Press, New York, 1980)

32. M Nakagami, The m-distribution, a general formula of intensity of rapid fading, in Statistical Methods in Radio Wave Propagation: Proceeding of a Symposium, June (Pergamon Press, Oxford, 1958)

33. JG Proakis, Digital Communications, 3rd ed. (McGraw-Hill, New York, 1995)

34. MK Simon, MS Alouini, A unified approach to the probability of error for noncoherent and differentially coherent modulations over generalized fading channels. IEEE Trans. Commun. 46(12), 1625-1638 (1998)

35. V Rotar, Probability and Stochastic Modeling. (Chapman and Hall/CRC, Boca Raton, 2012)

\section{Submit your manuscript to a SpringerOpen ${ }^{\odot}$ journal and benefit from:}

- Convenient online submission

- Rigorous peer review

- Immediate publication on acceptance

- Open access: articles freely available online

- High visibility within the field

- Retaining the copyright to your article

Submit your next manuscript at $\boldsymbol{\nabla}$ springeropen.com 